Cahiers

Furles Droit Fondamentaux
Cahiers de la recherche sur les droits fondamentaux

9| 2011

Conseil constitutionnel et droits fondamentaux

\title{
Chronique de jurisprudence de la Cour interaméricaine des Droits de l'homme
}

\section{Marie Rota}

\section{(2) OpenEdition}

\section{Journals}

Édition électronique

URL : https://journals.openedition.org/crdf/5485

DOI : $10.4000 /$ crdf.5485

ISSN : 2264-1246

Éditeur

Presses universitaires de Caen

\section{Édition imprimée}

Date de publication : 1 décembre 2011

Pagination : 155-169

ISBN : 978-2-84133-396-7

ISSN : 1634-8842

\section{Référence électronique}

Marie Rota, "Chronique de jurisprudence de la Cour interaméricaine des Droits de l'homme », Cahiers de la recherche sur les droits fondamentaux [En ligne], 9| 2011, mis en ligne le 01 décembre 2012,

consulté le 14 novembre 2022. URL : http://journals.openedition.org/crdf/5485 ; DOI : https://doi.org/ $10.4000 /$ crdf. 5485 


\section{Chronique de jurisprudence de la Cour interaméricaine des Droits de l'homme ${ }^{1}$}

I. L'obligation de respecter les droits (article 1.1)

II. Les droits protégeant les libertés physiques de la personne humaine

A. Les disparitions forcées: une violation «multiple » de droits

B. Droit à la vie (article 4)

C. Droit à l'intégrité (article 5)

1. Droit à l'intégrité d'une communauté indigène du fait de la privation de leurs terres

2. Violences sexuelles et droit à l'intégrité

3. Droit à l'intégrité des détenus

4. Droit à l'intégrité des membres de la famille de la victime

D. Protection de la vie privée, de l'honneur et de la dignité de la personne (article 11)

III. Les droits protégeant la liberté de la personne

A. Le droit à la liberté de la personne (article 7)

1. Le droit de ne pas être privé illégalement et arbitrairement de sa liberté (articles 7.2 et 7.3)

2. Le droit de connaître les raisons de son arrestation et des accusations portées contre soi (article 7.4)

3. Le contrôle judiciaire de la privation de liberté (article 7.5)

4. La possibilité de contester la légalité de la détention (article 7.6)

B. Le droit de déplacement et de résidence (article 22)

IV. Les droits protégeant la démocratie

A. La lecture combinée des droits politiques (article 23), de la liberté d'association (article 16) et de la liberté de pensée et d'expression (article 13)

B. Disparitions forcées et droits à la représentation politique (article 23.1.a)

C. Liberté de pensée et d'expression et droit à la vérité

V. Les droits protégeant les libertés relationnelles

L’année 2010 est marquée par de nombreux bouleversements institutionnels, qu'il s'agisse en Europe de l'entrée en vigueur inespérée du Protocole 14 de la Convention de sauvegarde des Droits de l'homme et des libertés fondamentales ${ }^{2}$, et outre-Atlantique, de l'entrée en vigueur du nouveau règlement ${ }^{3}$ de la Cour interaméricaine des

1. Chronique réalisée par Marie Rota, ATER à l'université du Havre, doctorante au CRDFED (Université de Caen Basse-Normandie), membre associé du GREDFIC (Université du Havre), du CEDIN (Centro de Direito Internacional - Brésil) et membre du GRIB (Groupe de recherche interdisciplinaire sur le Brésil).

2. Ci-après «la CSDH» ou «la Convention européenne».

3. Règlement adopté par la Cour lors de sa $85^{\circ}$ session ordinaire, 16-28 novembre 2009. 
Droits de l'homme ${ }^{4}$. La composition de cette dernière $\mathrm{a}$, par ailleurs, fait l'objet d'un renouvellement partiel, suite auquel un nouveau président est élu: le juge péruvien Diego García-Sayán ${ }^{5}$. Cette année de transition explique le ralentissement de l'activité juridictionnelle de la Cour, qui n'a adopté que neuf décisions au contentieux (contre dix-huit l'année dernière), portant sur le fond ${ }^{6}$.

Six décisions sont examinées suite à des reconnaissances étatiques de responsabilité, ce qui s'explique sans aucun doute par la nature du contentieux, plus «traditionnel ${ }^{7}$ que celui observé l'année précédente ${ }^{8}$. La Cour a, en effet, eu à traiter d'affaires révélatrices du passé dictatorial des États parties, comme en attestent les trois décisions portant sur le phénomène des disparitions forcées durant les anciennes dictatures militaires guatémaltèque, bolivienne et brésilienne ${ }^{9}$, mais s'est aussi attardée sur des sujets s'inscrivant dans un contexte politico-social plus contemporain. Deux décisions portent sur les droits des membres de communautés indigènes, la première sur la violation du droit de propriété ancestrale de la communauté Xákmok Kásek au Paraguay ${ }^{10}$, et la seconde sur des violences sexuelles commises par des militaires à l'encontre de femmes indigènes vivant dans l'État de Guerrero au Mexique, dans le contexte de la lutte des forces armées menée à l'encontre des narcotrafiquants ${ }^{11}$. La Cour s'attarde également sur l'arrestation et la détention par des militaires de Cabrera García et Montiel Flores dans cette même région du Mexique, ces derniers étant accusés d'avoir commis des délits de droit commun (usage d'armes et infraction à la législation sur les stupéfiants) ${ }^{12}$. L'affaire Manuel Cepeda Vargas c. Colombie traite, quant à elle, de l'exécution extrajudiciaire de ce sénateur dans un contexte de répression systématique des membres du parti communiste colombien (ci-après «le PCC») et de l'Union patriotique (ci-après «l'UP») durant la fin des années 1980 et le début des années $1990^{13}$. Seule l'affaire Vélez Loor c. Panama, relative à la violation de l'intégrité de ce migrant en situation irrégulière et détenu dans l'attente d'une mesure d'éloignement ${ }^{14}$, semble dès lors faire exception à ce constat.

On se contentera, comme dans notre précédente chronique, de retracer l'évolution de la protection juridictionnelle des droits tels que consacrés par la Convention américaine relative aux Droits de l'homme ${ }^{15}$. On ne s'attardera cependant pas sur les droits à la garantie et à la protection judiciaire (articles 8 et 25), la Cour ne faisant que réitérer ses principes généraux en la matière. On peut simplement souligner qu'elle rappelle certains aspects marquants de sa jurisprudence que nous avions relevés dans notre deuxième chronique ${ }^{16}$, à savoir la valeur de jus cogens qu'elle attache à l'obligation d'enquête face aux situations de disparitions forcées ${ }^{17}$; la portée de l'obligation d'enquête ex officio à partir du moment où l'État a connaissance d'une violation potentielle d'un droit protégé $^{18}$, ou encore celle du droit à la vérité ${ }^{19}$. Elle réaffirme en outre d'autres principes, issus de sa jurisprudence tant

4. Ci-après «la Cour» ou «la Cour de San José».

5. Juge à la Cour depuis 2004, vice-président depuis 2008, et réélu en 2010.

6. Contrairement aux années précédentes, la Cour n'a adopté aucune décision portant sur l'interprétation de décisions antérieures; elle a, en revanche, adopté 38 résolutions relatives aux mesures provisoires (disponibles sur www.corteidh.or.cr/medidas.cfm) et 40 résolutions relatives à la supervision de l'exécution de ses arrêts (disponibles sur www.corteidh.or.cr/supervision.cfm).

7. Voir M. Rota, «Chronique de jurisprudence de la Cour interaméricaine des Droits de l'homme», Cahiers de la recherche sur les droits fondamentaux, $\mathrm{n}^{\circ}$ 7, 2009, p. 189-198, ci-après «notre deuxième chronique»; "La Déclaration universelle des Droits de l'homme: source des droits garantis par la Convention américaine relative aux Droits de l'homme", Cahiers de la recherche sur les droits fondamentaux, ${ }^{\circ} 7,2009, \mathrm{p} .63-71$.

8. M. Rota, "Chronique de jurisprudence de la Cour interaméricaine des Droits de l'homme", Cahiers de la recherche sur les droits fondamentaux, $\mathrm{n}^{\circ} 8,2010$, p. 163-176, ci-après «notre précédente chronique».

9. Cour IDH, Chitay Nech et autres $c$. Guatemala, exceptions préliminaires, fond, réparations et frais, 25 mai 2010, série C, $\mathrm{n}^{\circ} 212$, ci-après «affaire Chitay Nech"; Cour IDH, Ibsen Cárdenas et Ibsen Peña c. Bolivie, fond, réparations et frais, $1^{\text {er }}$ septembre 2010, série C, $\mathrm{n}^{\circ} 217$, ci-après «affaire Ibsen"; et Cour IDH, Gomes Lund et autres (Guerrilha do Araguaia) c. Brésil, exceptions préliminaires, fond, réparations et frais, 24 novembre 2010, série C, $\mathrm{n}^{\circ}$ 219, ci-après «affaire de la Guerrilha do Araguaia».

10. Cour IDH, Communauté indigène Xákmok Kásek c. Paraguay, fond, réparations et frais, 24 août 2010, série C, $\mathrm{n}^{\circ}$ 214, ci-après «affaire Communauté indigène Xákmok Kásek».

11. Cour IDH, Fernández Ortega et autres c. Mexique, exceptions préliminaires, fond, réparations et frais, 30 août 2010 , série C, $\mathrm{n}^{\circ} 215$, ci-après "affaire Fernández Ortega»; et Cour IDH, Rosendo Cantú et autres c. Mexique, exceptions préliminaires, fond, réparations et frais, 31 août 2010 , série $\mathrm{C}, \mathrm{n}^{\circ}$ 21, ci-après «affaire Rosendo Cantú ».

12. Cour IDH, Cabrera García et Montiel Flores c. Mexique, exceptions préliminaires, fond, réparations et frais, 26 novembre 2010 , série $\mathrm{C}$, $\mathrm{n}^{\circ} 22 \mathrm{O}$, ci-après «affaire Cabrera García et Montiel Flores».

13. Cour IDH, Manuel Cepeda Vargas $c$. Colombie, exceptions préliminaires, fond, réparations et frais, 26 mai 2010, série $\mathrm{C}, \mathrm{n}^{\circ} 213$, ci-après « affaire Manuel Cepeda Vargas ". La Cour constate, en effet, qu'entre 1987 et 1993, les membres de ces partis étaient considérés comme «ennemis de l'intérieur», en vertu de la «doctrine de sécurité nationale» ( $\$ 85)$, fait reconnu tant au plan national qu'international ( $\$ 81)$, et qui a donné naissance à plusieurs "plans» dirigés contre les membres de ces partis dont l'« opération coup de grâce» visant entre autres la victime ( $\$ 86)$. Pour un aperçu du contexte de cette affaire, en langue française, voir également: I. Cepeda Castro et C. Girón Ortiz, «Vie et mort de l’Union patriotique. Comment des milliers de militants ont été liquidés en Colombie ", Le Monde diplomatique, mai 2005, disponible sur http://www.mondediplomatique.fr/2005/05/CEPEDA_CASTRO/12196.

14. Cour IDH, Vélez Loor c. Panama, exceptions préliminaires, fond, réparations et frais, 23 novembre 2010, série C, $\mathrm{n}^{\circ}$ 218, ci-après « affaire Vélez Loor ».

15. Ci-après «la CADH» ou «la Convention».

16. Voir notre deuxième chronique, p. 194.

17. Affaires Chitay Nech, $\$ 193$; Isben, $\$ 197$; de la Guerrilha do Araguaia, $\$ 137$; caractère reconnu dans l'affaire Cour IDH, Goiburú et autres c. Paraguay, fond, réparations et frais, 22 septembre 2006 , série $C, \mathrm{n}^{\circ} 153, \S 84$, ci-après «affaire Goiburú ».

18. Affaires Chitay Nech, $\$ 193$; Isben, $\$ 155 ;$ Fernández Ortega, $\$ 191 ;$ Rosendo Cantú, $\$ 175$; de la Guerrilha do Araguaia, $\$ 138$.

19. Affaires Chitay Nech, $\$ 207-209$; et Isben, $\$ 214-226$. 
contentieuse que consultative, tels que la nature des garanties que doivent présenter les juridictions militaires ${ }^{20}$, le caractère imprescriptible des "graves violations des Droits de l'homme ${ }^{21}$, le droit à l'assistance consulaire ${ }^{22}$ ainsi qu'à une assistance juridique gratuite ${ }^{23}$ dont doivent bénéficier les étrangers détenus ${ }^{24}$. Nous ne nous attarderons pas non plus sur le droit à la propriété communautaire des populations indigènes, la Cour ne faisant que réaffirmer sa jurisprudence en la matière, largement étudiée en doctrine francophone ${ }^{25}$.

Nous commencerons par aborder l'obligation générale à la charge des États parties de respecter les droits (I), ce qui nous permettra d'éclairer la méthodologie de la Cour concernant son interprétation des droits protégeant les libertés physiques (II), la liberté de la personne (III), et la démocratie (IV).

\section{L'obligation de respecter les droits (article 1.1)}

La Cour précise la portée de l'obligation de respecter et garantir les droits sans discrimination au travers des neuf décisions ${ }^{26}$. La Cour déduit de ce premier volet - la protection - que toute violation d'un droit entraîne systématiquement une violation combinée de cette disposition. Sa lecture combinée avec un autre droit de la Convention lui permet en outre de dégager tout un ensemble d'obligations positives à la charge des États. Nous pouvons à ce sujet souligner la méthodologie de la Cour qui s'appuie quasi systématiquement ${ }^{27}$ sur l'appartenance des victimes à des populations qu'elle qualifie de "vulnérables» : les populations indigènes ${ }^{28}$, particulièrement lorsqu'elles sont privées de leurs terres ${ }^{29}$ ou lorsqu'elles sont victimes d'un déplacement forcé en dehors de leur communauté ${ }^{30}$; les populations déplacées ${ }^{31}$; les victimes de disparitions forcées $^{32}$; les migrants ${ }^{33}$; les détenus ${ }^{34}$; les militants de l'UP et du PCC en Colombie ${ }^{35}$; et, enfin, la population de l'État de Guerrero au Mexique, confrontée à une forte présence militaire et policière sur ce territoire ${ }^{36}$. La Cour se fonde sur la lecture combinée des droits violés avec cet article 1.1 en ce qu'il interdit les discriminations, et qui a, d'après elle, valeur de jus cogens ${ }^{37}$. Elle estime que les États doivent non seulement s'abstenir d'agir de telle façon à ce qu'ils créent, "directement ou indirectement, des situations de jure ou de facto discriminatoires ${ }^{38}$, mais également adopter des mesures de protection spécifiques afin de les «prévenir et de [les] renverser ${ }^{39}$. Ceci implique

20. Affaires Fernández Ortega, $\$ 176-179$ et Rosendo Cantú, $\$ 160-163$. Voir K. Martin-Chenut, «La jurisprudence interaméricaine des Droits de l'homme sur l'exercice de la justice par les tribunaux militaires», Revue de science criminelle et de droit pénal comparé, $\mathrm{n}^{\circ} 2,2009, \mathrm{p} .442-453$.

21. Affaire Isben, $\$ 196-213$.

22. Affaire Vélez Loor, $\$ 151-160$; droit issu de la lecture combinée des articles 7.4, 8.1 et 8.2.d de la Convention.

23. Affaire Vélez Loor, \$141-148.

24. Cour IDH, Condition juridique et droits des travailleurs migrants, opinion consultative $\mathrm{n}^{\circ} \mathrm{OC}-18 / 03,17$ septembre 2003 , série $\mathrm{A}, \mathrm{n}^{\circ} 18$, ci-après "avis sur la Condition juridique et droits des travailleurs migrants».

25. C. Malwé, «La protection du droit de propriété par la Cour interaméricaine des Droits de l’homme», RTDH, n 78, 2009, p. 569-605; K. Rinaldi, «Le droit des populations autochtones et tribales à la propriété dans le système interaméricain de protection des Droits de l'homme», in Le particularisme interaméricain des Droits de l'homme. En l'honneur du 40 anniversaire de la Convention américaine des Droits de l'homme (1969-20o9), L. Hennebel et H. Tigroudja (dir.), Paris, Pedone, 2009, p. 214-250; L. Hennebel, «La protection de l'“intégrité spirituelle” des indigènes. Réflexions sur l'arrêt de la Cour interaméricaine des Droits de l'homme dans l'affaire Communidad Moiwana c. Suriname du 15 juin 2005 ", RTDH, ${ }^{\circ}$ 66, 2006, p. 263-265; L. Hennebel et H. Tigroudja, «Chronique des décisions rendues par la Cour interaméricaine des Droits de l’homme (20062007) ", RTDH, $\mathrm{n}^{\circ}$ 76, 2008, p. 1038-1039; L. Hennebel, La Convention américaine des Droits de l'homme, Bruxelles, Bruylant, 2007, p. 612-614.

26. En ce qui concerne la seconde obligation générale, l'article 2, qui met à la charge des États une obligation de mise en conformité de leur droit interne avec ses dispositions, on peut simplement mentionner que la Cour considère que la législation relative au droit des étrangers du Panama, l'article 57.II.a du Code de justice militaire mexicain prévoyant une compétence générale des tribunaux militaires, et la loi d’amnistie brésilienne ayant entravé l'enquête sur la disparition forcée de la victime, sont tous trois incompatibles avec la Convention, d'où une violation constatée de cette disposition: affaires Vélez Loor, $\$ 194$; Fernández Ortega, $\$ 178-179$; Rosendo Cantú, \$162-163; et de la Guerrilha do Araguaia, $\$ 171-174$.

27. Seule l'affaire de la Guerrilha do Araguaia n'y fait pas référence.

28. Affaires Communauté indigène Xákmok Kásek, \$270; Fernández Ortega, \$201; et Rosendo Cantú, \$185.

29. Affaire Communauté indigène Xákmok Kásek, $\$ 190$.

30. Affaire Chitay Nech, $\$ 147$. Il est intéressant de noter que pour motiver cette approche, la Cour se fonde cette fois-ci sur le droit interne d'un État membre, à savoir la décision de la Cour constitutionnelle colombienne du 26 janvier 2009, Auto o04/o09.

31. Affaire Chitay Nech, $₫ 141$; Cour IDH, Massacre de Mapiripán c. Colombie, fond, réparations et frais, 15 septembre 2005 , série C, $\mathrm{n}^{\circ}{ }_{134}, \S 210$, ci-après «affaire Massacre de Mapiripán».

32. Affaires Chitay Nech, $\$ 96$; et Isben, $\$ 99$.

33. Affaire Vélez Loor, $\$ 97-101$. Cour IDH, Bayarri c. Argentine, exceptions préliminaires, fond, réparations et frais, 30 octobre 2008, série C, ${ }^{\circ}$ 187, $\$ 67$, ci-après «affaire Bayarri». La Cour rappelle à cet égard tous les principes qu'elle avait posés dans son avis protecteur. Voir avis sur la Condition juridique et droits des travailleurs migrants, $\$ 117$, repris par différents rapports de l'ONU, allant dans le même sens et cités par la Cour. Voir également, dans le même sens, l'arrêt de la CEDH, Iwanczuk c. Pologne, 15 novembre 2001, requête n ${ }^{\circ} 25196 / 94, \$ 53$.

34. Affaire Vélez Loor, $\$ 207$.

35. Affaire Cepeda Vargas, $\$ 81-86$

36. Affaire Cabrera García et Montiel Flores, $\$ 86$.

37. La Cour reconnaît cette valeur depuis son avis sur la Condition juridique et droits des travailleurs migrants, $\$ 101$ et l'affaire Yatama c. Nicaragua, exceptions préliminaires, fond, réparations et frais, 23 juin 2005, série $\mathrm{C}, \mathrm{n}^{\circ} 127, \$ 184$, ci-après «affaire Yatama ». Il est dès lors remarquable que la Cour cite en plus 34 instruments internationaux protégeant cet aspect (instruments de l'OEA, suivis de ceux des Nations unies, et, pour finir, des autres instruments régionaux) : affaire Communauté indigène Xákmok Kásek, \$269.

38. Affaire Communauté indigène Xákmok Kásek, $\$ 271$. Voir avis sur la Condition juridique et droits des travailleurs migrants, $\$ 103$.

39. Affaire Communauté indigène Xákmok Kásek, \$271. Voir également l'affaire Chitay Nech, \$147. 
l'obligation de protéger ces groupes à l'égard des actes ou des pratiques des tiers, dans la mesure où l'abstention de l'État aura pour effet de «favoriser, encourager, promouvoir ou consolider leur vulnérabilité ${ }^{40}$.

Enfin, dans l'affaire Communauté indigène Xákmok Kásek, la Cour consacre toute une partie de sa décision à la violation spécifique de cet article 1.1, alors qu'elle effectuait uniquement jusque-là une lecture combinée de cette disposition avec les droits protégés. Elle commence par rappeler que les États doivent accorder aux populations indigènes une protection particulière, «qui tienne compte de leurs spécificités, de leurs caractéristiques économiques et sociales, ainsi que de leur état de particulière vulnérabilité, de leur droit coutumier, de leurs valeurs, usages et coutumes» $(\$ 270)^{41}$. Or, l'État a manqué à cette obligation concernant l'exercice des droits dont elle a constaté la violation; cette communauté se trouve «marginalisée dans la jouissance de ses droits » (\$274); elle est, dès lors, dans un état de "discrimination de facto", d'où une violation de l'article 1.1. Mais ce n'est que parce que d'autres droits ont été violés qu'elle aboutit à cette conclusion. Il ne s'agit donc pas d'une autonomisation totale de cette disposition, même si cette démarche l'annonce peut-être ${ }^{42}$.

\section{Les droits protégeant les libertés physiques de la personne humaine}

Nous commencerons par aborder l'analyse que fait la Cour des droits protégeant les libertés physiques face à la pratique des disparitions forcées (1), et nous nous attarderons ensuite sur la protection juridictionnelle du droit à la vie (2), du droit à l'intégrité (3), et du droit à la protection de la vie privée, de l'honneur et de la dignité de la personne (4).

\section{A. Les disparitions forcées: une violation « multiple » de droits}

La Cour constate dans les trois affaires Chitay Nech, Ibsen, et de la Guerrilha do Araguaia que les États se sont rendus responsables de disparitions forcées ${ }^{43}$. C'est l'occasion pour la Cour de réaffirmer les caractéristiques principales que recouvre, selon elle, cette pratique, qui s'analyse en une violation "grave», "autonome», "permanente» et «multiple» de la Convention, ce qui l'empêche d'apprécier isolément la violation de chaque droit ${ }^{44}$. Ceci justifie qu'elle applique une fois encore son «mécanisme déductif » ${ }^{45}$ pour conclure à la violation des droits à la liberté individuelle (article 7), à l'intégrité personnelle (article 5), à la vie (article 4) et à la reconnaissance de la personnalité juridique (article 3$)^{46}$, alors même qu'aucune preuve d'atteinte à ces trois derniers droits n'aurait été apportée.

Cette façon de procéder appelle deux remarques. La première s'attache à la définition et aux caractéristiques des disparitions forcées. Bien qu'elles correspondent à celles que la Cour a déjà ultérieurement dégagées ${ }^{47}$, elle semble ressentir le besoin de légitimer encore et toujours cette approche au regard de normes exogènes. Elle cite ainsi les définitions contenues dans divers instruments internationaux, dont elle ne cesse d'enrichir la liste ${ }^{48}$, et, chose qu'elle ne faisait pas auparavant, de différentes «cours constitutionnelles des États américains et hauts tribunaux nationaux», qui coïncident avec la sienne ${ }^{49}$. Ceci lui permet de souligner «la consolidation au niveau international de la définition du crime de disparition forcée» et de réaffirmer que sa prohibition a «atteint le caractère de jus cogens ${ }^{50}$.

La seconde remarque concerne les droits considérés comme étant atteints par ce crime. Comme nous l'avions remarqué lors de notre deuxième chronique, la Cour

40. Affaires Communauté indigène Xákmok Kásek, $\$ 271$; Chitay Nech, $\$ 147$; et Manuel Cepeda Vargas, $\$ 172$. Voir avis sur la Condition juridique et droits des travailleurs migrants, $\$ 112$ et 172 .

41. Voir Cour IDH, Communauté indigène Yakye Axa c. Paraguay, fond, réparations et frais, 17 juin 2005, série $\mathrm{C}, \mathrm{n}^{\circ} 125, \S 63 ;$ ci-après «affaire Communauté indigène Yakye Axa».

42. Sur ce point, voir L. Hennebel, La Convention américaine des Droits de l'homme, Bruxelles, Bruylant, 2007, p. 358-359.

43. La Cour rappelle que la disparition forcée s'analyse en une privation de liberté rendue possible du fait de l'intervention directe des agents de l'État, ou issue de son consentement, et caractérisée par le refus étatique de reconnaître cette détention et de révéler le sort de la personne concernée ou le lieu où elle se trouve. Affaire Chitay Nech, $\$ 85$.

44. Affaires Chitay Nech, $\$ 81-88 ;$ Isben, $\$ 57-68$; et de la Guerrilha do Araguaia, $\$ 101-111$.

45. Voir notre deuxième chronique, p. 195; J. Benzimra-Hazan, «Disparitions forcées de personnes et protection du droit à l’intégrité: la méthodologie de la Cour interaméricaine des Droits de l'homme», $R T D H, \mathrm{n}^{\circ} 47,2001, \mathrm{p} .765-796$.

46. Articles lus conjointement avec l'article 1.1 de la CADH et les articles 1 et 11 de Convention interaméricaine sur les disparitions forcées.

47. Tant la définition que les caractéristiques de la disparition forcée ressortent de sa jurisprudence traditionnelle: voir, par exemple, l'affaire Cour IDH, Gómez Palomino c. Pérou, fond, réparations et frais, 22 novembre 2005, série C, $\mathrm{n}^{\circ} 136 \$ 97$ pour la définition, et affaire Goiburú, $\$ 83$ pour les caractéristiques des disparitions forcées.

48. La Cour se réfère comme à son habitude à l'article 2 de la Convention internationale pour la protection de toutes les personnes contre les disparitions forcées (affaires Chitay Nech, $\$ 83$; Ibsen, $\$ 60$; et de la Guerrilha do Araguaia, $\$ 104$ ), mais aussi au Statut de Rome (affaire Chitay Nech, $\$ 85$ ), au rapport du Groupe de travail sur les disparitions forcées ou involontaires de personnes du Conseil économique et social de l'Organisation des Nations unies (affaires Chitay Nech $\$ 82$; Isben, $\$ 60$; et de la Guerrilha do Araguaia, $\$ 104$ ), et aux décisions du Comité des Droits de l'homme des Nations unies (affaires Chitay Nech, $\$ 85$; Isben, $\$ 60$; et de la Guerrilha do Araguaia, $\$ 104$ ). La Cour cite aussi la jurisprudence européenne s'étant elle-même inspirée de la méthodologie interaméricaine à l'égard des disparitions forcées: affaires Chitay Nech, $\$ 85$; Isben, $\$ 60$; et de la Guerrilha do Araguaia, $\$ 104$.

49. La Cour cite les définitions nationales dégagées par les tribunaux vénézuélien, mexicain, chilien, argentin, bolivien, péruvien et uruguayen affaires Chitay Nech, $\$ 60$; et de la Guerrilha do Araguaia, $\$ 104$.

50. Affaires Chitay Nech, $\$ 86$; Isben, $\$ 61$; et de la Guerrilha do Araguaia, $\$ 105$. Voir également l'affaire Goiburú, $\$ 59$. 
commence systématiquement par caractériser l'atteinte à la liberté individuelle, et, eu égard au contexte, en déduit une atteinte aux droits à la vie et à l'intégrité. Elle estime dorénavant qu'une disparition forcée emporte également une violation de l'article 3 de la Convention, chose qu'elle a faite la première fois en $2009^{51}$, et qu'elle prend soin de confirmer dans ces trois espèces. Ceci explique l'attitude du Guatemala dans l'affaire Chitay Nech, qui reconnaît sa responsabilité concernant les trois premiers droits, mais s'oppose à ce que ces allégations de disparitions forcées soient examinées sous l'angle de ce nouvel article ${ }^{52}$. La Cour prend alors soin de justifier cette approche, en s'appuyant sur «le principe de l'effet utile», lui imposant de prendre en considération «l'évolution du corpus juris international» en la matière ${ }^{53}$.

\section{B. Droit à la vie (article 4)}

Dans l'affaire Communauté indigène Xákmok Kásek, la Cour relève que le droit à la vie est un droit humain fondamental, dont la pleine jouissance est une condition préalable à la jouissance de tous les autres droits ${ }^{54}$. C'est la raison pour laquelle elle déduit de la lecture combinée de l'article 4 avec l'article 1.1 de la Convention trois séries d'obligations : la première impliquant que les États ne peuvent priver personne arbitrairement de sa vie (obligation négative), la deuxième leur imposant de prendre toutes les mesures appropriées pour protéger et préserver le droit à la vie (obligation positive) ${ }^{55}$, la troisième de mener une enquête ex officio et effective sur la violation du droit à la vie (obligation positive procédurale). C'est au titre de ces deux dernières que le Paraguay et la Colombie (dans l'affaire Manuel Cepeda Vargas) sont reconnus responsables d'une violation du droit à la vie.
Dans les deux espèces, qui concernent des populations indigènes d'une part et le sénateur d'autre part, la Cour identifie l'existence d'une situation de risque d'atteinte à la vie connu des autorités ${ }^{56}$, qui fait naître à leur égard un devoir spécifique de prévention ${ }^{57}$ et de protection $^{58}$. Or, ils ne sont en l'espèce pas respectés, du fait de l'inaction de la Colombie dans la seconde affaire et de l'insuffisance des moyens mis en œuvre par le Paraguay dans la première, d'où une violation de l'article 4.1 de la Convention.

On peut ici relever les différentes composantes du «droit de mener une vie digne», que la Cour inclut dans cette obligation de prévention, faisant entrer tout un ensemble de droits économiques et sociaux dans le champ de la Convention. Il s'agit de l'accès à l'eau, à l'alimentation, à la santé et à l'éducation ${ }^{59}$ appréciés au regard des standards internationaux ${ }^{60}$. Relevant qu'aucun d'entre eux n'est respecté, la Cour constate que cette situation empêche les membres de la communauté Xákmok Kásek de vivre selon leurs coutumes et les amène à vivre dans la misère ${ }^{61}$. Le Paraguay n'a donc pas respecté son obligation de protection du droit de mener une vie digne, au détriment de l'ensemble de ses membres ( $\$ 217)$.

Mais le raisonnement de la Cour ne s'arrête pas là. Dans l'affaire Manuel Cepeda Vargas, elle constate que les États confrontés à de graves violations des Droits de l'homme, telles que les cas d'exécutions extrajudiciaires, entre autres, ont l'obligation de mener " une enquête ex officio, sans délai et de manière sérieuse, impartiale et efficace», qui « doit viser la poursuite, l'arrestation, la traduction en justice et la sanction éventuelle de tous les auteurs, surtout lorsque des agents de l'État peuvent être impliqués» $(\$ 117)^{62}$. Or, et du fait du manque de cohérence et de l'insuffisance des enquêtes menées au plan interne (\$120-122), elle estime que la Colombie a également violé

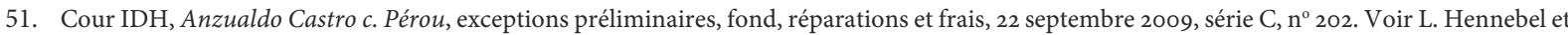
H. Tigroudja, «Chronique des décisions rendues par la Cour interaméricaine des Droits de l'homme (2008-2009)», RTDH, $\mathrm{n}^{\circ}$ 84, 2010, p. 830-831.

52. Affaire Chitay Nech, $\$ 80$; la Cour avait en effet refusé dans des affaires antérieures de reconnaître une violation de l'article 3 du fait d'une disparition forcée, voir Cour IDH, Bámaca Velásquez c. Guatemala, fond, 25 novembre 2000, série C, $\mathrm{n}^{\circ} 70, \$ 180$.

53. Corpus non identifié dans l'affaire Chitay Nech, mais qui l'était dans l'affaire Cour IDH, Anzualdo Castro $c$. Pérou, exceptions préliminaires, fond, réparations et frais, 22 septembre 2009, série $\mathrm{C}, \mathrm{n}^{\circ} 20, \$ 92-101$, à laquelle elle fait référence. Dans l'affaire de la Guerrilha do Araguaia, la Cour se contente de faire un résumé de son approche globalisante.

54. Affaire Communauté indigène Xákmok Kásek, $\$ 186$. Cette valeur a déjà été reconnue précédemment dès l'affaire des enfants des rues, Cour IDH, «Niños de la Calle» (Villagrán Morales et autres) c. Guatemala, fond, 19 novembre 1999, série C, nº 63, 144.

55. Affaire Communauté indigène Xákmok Kásek, $\$ 188$

56. Affaires Communauté indigène Xákmok Kásek, $\$ 190$; et Manuel Cepeda Vargas, $\$ 81-86$. Un tel contexte était connu des autorités du fait des différentes actions menées au plan interne dans la première affaire; dans la seconde, le sénateur a dénoncé ce plan en 1993 devant le ministère de la Défense $(\$ 89)$; il en a averti le Congrès $(\$ 92)$ et a fait une demande de protection de la part de l'État, jamais accordée ( $\$ 94)$. La Cour constate en outre que plusieurs personnes ont effectivement été victimes de ce «plan», dont le sénateur en 1994 ( $\$ 98)$.

57. Affaire Communauté indigène Xákmok Kásek, \$192.

58. Affaire Manuel Cepeda Vargas, \$101.

59. Affaire Communauté indigène Xákmok Kásek, $\$ 195-211$.

60. La Cour se réfère à l'article 13.3.a du Protocole de San Salvador traitant des droits économiques sociaux et culturels du 17 novembre 1988 ; au Comité des droits économiques, sociaux et culturels des Nations unies, Observation générale $n^{\circ} 12,12$ mai 1999, E/C.12/1999/5, $\$ 6$, Observation générale $n^{\circ} 13,8$ décembre 1999, E/C.12/1999/10, \$50, Observation générale $n^{\circ} 15,11-29$ novembre 2002, E/C.12/2002/11, \$12, et Observation générale $n^{\circ} 21,21$ décembre 2009, E/C.12/GC/21, $\$ 38$; à la Convention $n^{\circ} 169$ de l'Organisation internationale du travail concernant les peuples indigènes et tribaux dans les pays indépendants du 27 juin 1989; au rapport de J. Bartram et G. Howard, «La cantidad de agua domiciliaria, el nivel de servicio y la salud», OMS, 2002, WHO/SDE/WSH/03.02; mais aussi à l'article de P. H. Gleick, «Basic water requirements for human activities: meeting basic needs", Water International, $\mathrm{n}^{\circ} 21,1996$, p. 83-92.

61. La Cour fait encore une fois référence au Comité des droits économiques, sociaux et culturels des Nations unies pour définir cette notion: Communauté indigène Xákmok Kásek, \$215.

62. Voir Cour IDH, Massacre Pueblo Bello c. Colombie, fond, réparations et frais, 31 janvier 2006, série C, $\mathrm{n}^{\circ} 140, \$ 143$ et 290. 
son obligation positive procédurale issue de la lecture combinée des articles 4 et 1.1 de la Convention.

\section{Droit à l'intégrité (article 5)}

La Cour est confrontée en 2010 à plusieurs violations du droit à l'intégrité du fait de la privation des terres des populations indigènes (a), de viols (b), ou encore de la situation de détenus (c). Elle confirme, en outre, que la violation de certains droits de la victime principale peut avoir des répercussions sur l'intégrité des membres de sa famille (d).

\section{Droit à l'intégrité d'une communauté indigène du fait de la privation de leurs terres}

Dans l'affaire Communauté indigène Xákmok Kásek, la Cour rappelle que la séparation de leurs terres traditionnelles ${ }^{63}$ peut entraîner des souffrances telles qu'elles s'analysent en une violation de l'article 5.1 (\$243). En l'espèce, la Cour souligne «la perte graduelle de la culture» de ces populations, leurs conditions de vie, le décès de plusieurs de leurs membres, accompagné d'une situation d'impunité, ainsi que l'état général d'abandon dans lequel elles vivent. Elle estime par conséquent que les souffrances induites «atteignent nécessairement l'intégrité mentale et morale de tous les membres de la communauté », d'où une violation de l’article 5.1 à leur égard (\$244).

\section{Violences sexuelles et droit à l'intégrité}

La Cour examine deux cas de viols commis par des militaires au regard des exigences de l'article 5.1 de la Convention dans les deux affaires jumelles Fernández Ortega et Rosendo Cantú ${ }^{64}$. Pour déterminer si le viol peut être qualifié d'un acte de torture, la Cour se réfère à sa jurisprudence antérieure ${ }^{65}$ et à la définition de la Convention interaméricaine pour la prévention et la répression de la torture ( $\$ 120$ et 110) et réaffirme les trois critères nécessaires à cette qualification. Il s'agit tout d'abord de l'inten- tionnalité de l'acte et la Cour relève que le viol a bien été commis de manière délibérée ${ }^{66}$. Concernant la «souffrance physique et mentale sévère engendrée ${ }^{67}$, ensuite, la Cour affirme que ce critère est toujours considéré comme étant rempli s'agissant d'un viol, et qu'elle est en outre aggravée au vu des circonstances des deux espèces: ils ont été commis devant d'autres militaires ( $\$ 125$ et 115$)$, sur une mineure dans la seconde affaire $(\$ 115)$, en présence des enfants de la victime dans la première, et ils ont engendré sa "perte d'esprit », telle qu'elle ressort de la tradition indigène $(\$ 125-126)$. Concernant, enfin, le «but ou l'intention particulière» des auteurs de l'acte, qui peut être « d'intimider, dégrader, humilier, punir ou de contrôler la personne qui souffre», la Cour constate que ces actes ont bien été commis en représailles, face à l'impossibilité des victimes de fournir aux militaires certaines informations, celles-ci ne parlant pas la même langue ( $\$ 127$ et 117 ). Le Mexique est, par conséquent, reconnu responsable d'une violation des articles 5.1 et 1.1 de la Convention.

\section{Droit à l'intégrité des détenus}

La Cour de San José accorde une protection spécifique aux détenus ${ }^{68}$, considérés comme "groupe vulnérable». Dans l'affaire Vélez Loor, elle dresse un bilan de sa jurisprudence en la matière, rappelant que, conformément aux articles 5.1 et 5.2 de la Convention, "chaque détenu a le droit de vivre dans des conditions de détention compatibles avec sa dignité personnelle» (\$198), les États étant compétents pour garantir les droits de toute personne se trouvant sous sa garde ${ }^{69}$. Ils ont à leur charge différentes obligations positives de protection, tant de la santé que du bien-être des détenus, et doivent s'assurer que les conditions d'incarcération "ne dépassent pas le niveau inévitable de souffrance inhérent à la détention ${ }^{70}$.

Concernant le cas particulier de la privation de liberté des migrants en attente d'une mesure d'éloignement, la Cour précise qu'ils se trouvent dans une situation de «vulnérabilité accrue» lorsqu'ils sont détenus dans les mêmes locaux que des personnes prévenues ou condamnées $(\$ 207)^{71}$. Elle impose alors aux États de prévoir des

63. Cour IDH, Comunidad Moiwana c. Suriname, exceptions préliminaires, fond, réparations et frais, 15 juin 2005, série C, $\mathrm{n}^{\circ}$ 124, $\$ 101-103$, ci-après "affaire Communauté Moiwana».

64. La Cour cite à cet égard le préambule de la Convention de Belém do Pará, en ce qu'il affirme que «la violence contre la femme constitue une offense à la dignité humaine», qui «touche tous les secteurs de la société [...] et a des incidences sur ses bases mêmes». Affaires Fernández Ortega, $\$ 118$; et Rosendo Cantú, $\$ 108$.

65. Cour IDH, Buenos Alves c. Argentine, fond, réparations et frais, 11 mai 2007, série C, $\mathrm{n}^{\circ} 164, \$ 79$.

66. Affaires Fernández Ortega, $\$ 121$; et Rosendo Cantú, $₫ 111$.

67. Cette dernière pouvant consister en un acte de violence physique mais également de souffrance morale ou psychique aigue: Cour IDH, Cantoral Benavides c. Pérou, fond, 18 août 200o, série C, n 69, $\$ 100$, ci-après «affaire Cantoral Benavides». Affaires Fernández Ortega, $\$ 124$; et Rosendo Cantú, $₫ 114$.

68. Voir L. Burgorgue-Larsen, "Commentaire $\mathrm{n}^{\circ} 18$ sous l'arrêt Tibi », in Les grandes décisions de la Cour interaméricaine des Droits de l’homme, L. Burgorgue-Larsen et A. Úbeda de Torres (dir.), Bruxelles, Bruylant, 2008, p. 498-532.

69. Voir Cour IDH, Neira Alegría et autres c. Pérou, fond, 19 janvier 1995, série C, nº 20, $\$ 60$.

70. Voir Cour IDH, «Instituto de Reeducación del Menor» c. Paraguay, exceptions préliminaires, fond, réparations et frais, 2 septembre 2004 , série $\mathrm{C}, \mathrm{n}^{\circ} 112, \$ 159$, ci-après «affaire Instituto de Reeducación del Menor». À défaut, l'État se rend coupable d'une violation de l'interdiction absolue de commettre des actes de torture ou traitements cruels, inhumains ou dégradants: affaire Cantoral Benavides, $\$ 95$. La Cour cite les Observations finales du Comité contre la torture, qui vont dans le même sens: Observations finales, $25^{\mathrm{e}}$ session, 13-24 novembre 2000, A/56/44, 10 mai 2001, $\$ 95 \mathrm{f}$.

71. Pour justifier cette approche, la Cour cite notamment le rapport de la rapporteuse spéciale du Conseil économique et social des Nations unies, G. Rodríguez Pizarro, Groupes et individus particuliers: les travailleurs migrants, $1^{\mathrm{er}}$ novembre 2002, E/CN.4/2003/85, ci-après « rapport Rodríguez Pizarro». 
établissements distincts, en se fondant tant sur la qualité des deux groupes de personnes - migrants en situation irrégulière / prévenus et détenus - que sur les finalités de la détention - « le redressement et la réinsertion sociale» pour ces derniers, et la procédure d'éloignement pour les premiers. La Cour relève à cet égard tout un ensemble de normes internationales, plaidant en faveur de la reconnaissance de ce "principe de séparation ${ }^{72}$, dont elle prend acte ( $\$ 208)$. Mais elle précise aussi la portée de cette obligation, qui impose à l'État «d'adopter certaines mesures positives, concrètes et ciblées », «afin de prévenir le risque accru d'atteinte aux droits, à l'intégrité personnelle et familiale et au bien-être des migrants » (\$209). Constatant pour finir que la victime a été placée successivement dans une maison d'arrêt puis dans un centre pénitentiaire, la Cour reconnaît le Panama responsable d'une violation des articles 5.1 et 5.2, lus de manière combinée avec son article 1.1.

La Cour s'attarde ensuite sur les conditions de détention subies par la victime et rappelle, dans ce cadre, deux obligations positives à la charge de l'État vis-à-vis des détenus $^{73}$ : il doit permettre l'accès à l'eau potable $(\$ 215)^{74}$ et prévoir des examens médicaux réguliers et des soins ou traitements appropriés si nécessaire $(\$ 216)^{75}$. Néanmoins, malgré ses efforts, le Panama n'a pas respecté ces deux obligations et s'est rendu coupable d' « un traitement cruel, inhumain et dégradant, contraire à la dignité de l'être humain» (\$227).

Enfin, les États ont à leur charge une obligation positive procédurale issue de la lecture combinée des articles 5.1, 5.2 de la Convention avec son article 1.1, mais aussi avec les articles 1, 6 et 8 de la Convention interaméricaine pour la prévention et la répression de la torture. Ils doivent dès lors procéder à une enquête ex officio lorsqu'un acte de torture ou des traitements cruels, inhumains ou dégradants ont été, ou risquent d'être commis ${ }^{76}$. En l'espèce, l'enquête menée suite à une plainte de la victime n’ayant pas été menée avec les diligences nécessaires, le Panama a violé l'ensemble de ces dispositions (\$230-245).

\section{Droit à l'intégrité des membres de la famille de la victime}

La Cour a eu une fois encore l'occasion de réaffirmer que la violation de certains droits de la victime principale pouvait engendrer la violation du droit à l'intégrité de "victimes secondaires ${ }^{77}$, membres de sa famille ou amis ${ }^{78}$. Réaffirmant l'existence d'une présomption simple d'une telle violation à l'égard des membres de la famille directe (parents, enfants, époux, compagnons permanents) ${ }^{79}$, elle constate qu'elle n'a pas été renversée par les États dans les quatre espèces concernées ${ }^{80}$. La Cour précise ensuite quels sont les éléments qu'elle peut prendre en considération pour savoir si le droit à l'intégrité d'autres personnes est également atteint ${ }^{81}$ et reconnaît comme étant victimes les frères et sœurs dans les affaires Chitay Nech $(\$ 220-226)$ et de la Guerrilha do Araguaia (\$235-244), mais aussi d'un neveu dans cette dernière, et la nouvelle épouse du père de la victime dans l'affaire Isben ( $\$ 129-133)$.

\section{Protection de la vie privée, de l'honneur et de la dignité de la personne (article 11)}

Dans les affaires Fernández Ortega et Rosendo Cantú, la Cour précise la portée de l'article 11 qui protège la vie privée $(\$ 129 / 119)^{82}$, notion qu'elle appréhende par renvoi à

72. La Cour cite la jurisprudence de sa consœur européenne (CEDH, Zhu c. Royaume-Uni, recevabilité, 12 septembre 2000, requête $\left.\mathrm{n}^{\circ} 36790 / 97\right)$; mais aussi divers rapports de l'OEA, des Nations unies et du Comité européen pour la prévention de la torture et des peines ou traitements inhumains ou dégradants.

73. La Cour se réfère au Comité des droits économiques, sociaux et culturels des Nations unies, Observation générale $n^{\circ}$ 15, 11-29 novembre 2002, E/C.12/2002/11, ainsi qu'aux résolutions de l'assemblée de l'Organisation des États américains (Résolution portant sur l'eau, la santé et les Droits de l'homme, 5 juin 2007, AG/RES. 2349, XXXVII-O/o7), de l'assemblée générale des Nations unies (Résolution 64/292 relative au droit fondamental à l'eau et à l'assainissement, 28 juillet 2010, A/Res/64/292) et à l'Ensemble de règles minima pour le traitement des détenus adoptées par le premier Congrès des Nations unies pour la prévention du crime et le traitement des délinquants, tenu à Genève en 1955 et approuvé par le Conseil économique et social dans ses résolutions 663 C (XXIV) du 31 juillet 1957 et 2076 (LXII) du 13 mai 1977.

74. Voir également l'affaire Instituto de Reeducación del Menor, $\$ 152$. Elle estime en effet que sans eau, les détenus se trouvent privés de leur liberté de satisfaire un certain nombre de besoins fondamentaux, considérés comme essentiels pour le développement d'une vie digne, voir Cour IDH, Tibi c. Équateur, exceptions préliminaires, fond, réparations et frais, 7 septembre 2004, série $\mathrm{C}, \mathrm{n}^{\circ} 114, \$ 56$, ci-après «affaire Tibi».

75. La Cour insiste à cet égard sur l'indépendance des médecins vis-à-vis des autorités pénitentiaires, "garantie importante contre la torture et les mauvais traitements, physiques ou mentaux, des personnes privées de liberté» (affaire Vélez Loor, $\$ 224$ ). Voir également Cour IDH, Montero Aranguren et autres (Retén de Catia) c. Venezuela, exceptions préliminaires, fond, réparations et frais, 5 juillet 2006 , série $\mathrm{C}$, $\mathrm{n}^{\circ} 150, \$ 102$, ciaprès «affaire Retén de Catia».

76. Voir supra.

77. Affaires Vélez Loor, $\$ 220$ et 126 ; de la Guerrilha do Araguaia, $\$ 235$; Manuel Cepeda Vargas, $\$ 196$; et Chitay Nech, $\$ 220$. Sur ce point, voir les considérations de L. Burgorgue-Larsen, "Commentaire $\mathrm{n}^{\circ} 15$ sous l'arrêt Caesar c. Trinité et Tobago ", in Les grandes décisions de la Cour interaméricaine des Droits de l’homme, p. 428. Cour IDH, Castillo Páez c. Pérou, fond, 3 novembre 1997, série C, $\mathrm{n}^{\circ} 34$, point résolutif $\mathrm{n}^{\circ} 4$.

78. Affaires Chitay Nech, $\$ 220$; Isben, $\$ 126$; et de la Guerrilha do Araguaia, $\$ 235$. Voir Cour IDH, Valle Jaramillo et autres c. Colombie, exceptions préliminaires, fond, réparations et frais, 27 novembre 2008 , série $\mathrm{C}, \mathrm{n}^{\circ} 192, \$ 119$, ci-après «affaire Valle Jaramillo ».

79. Affaires Chitay Nech, $\$ 220 ;$ Isben, $\$ 127$; de la Guerrilha do Araguaia, $\$ 235$; et Fernández Ortega, $\$ 151$. Voir aussi l'affaire Valle Jaramillo, $\$ 119$.

80. Affaires Isben, $\$ 133$; Rosendo Cantú, $\$ 137$ et 139; Manuel Cepeda Vargas, $\$ 196$; et de la Guerrilha do Araguaia, $\$ 242$.

81. La proximité des personnes avec la victime, le fait qu'elles aient entrepris des recherches pour la retrouver en cas de disparition forcée, l'existence de souffrances causées par la violation des droits de la victime principale et l'inaction de l'État, etc.: affaires Isben, \$127; et de la Guerrilha do Araguaia, $\$ 238$.

82. Voir également: Cour IDH, Massacre de Ituango c. Colombie, exceptions préliminaires, fond, réparations et frais, $1^{\mathrm{er}}$ juillet 2006 , série C, $\mathrm{n}^{\mathrm{o}} 148$, $\$ 193$. 
la jurisprudence de la Cour européenne ${ }^{83}$. Elle considère alors qu'elle doit s'interpréter largement et qu'elle protège, entre autres, la vie sexuelle et le droit d'établir et de développer des relations avec d'autres êtres humains. Or le viol des victimes a atteint l'un des «aspects essentiels de leur vie privée» $(\$ 129 / 119)^{84}$, constitutif d'une atteinte à l'article 11.

\section{Les droits protégeant la liberté de la personne}

Durant cette même période, la Cour se penche sur deux droits protégeant la liberté de la personne: le droit à la liberté (1) et le droit de déplacement et de résidence (2).

\section{A. Le droit à la liberté de la personne (article 7)}

La Cour a eu l'occasion de préciser la portée générale de l'article 7 de la Convention au travers des décisions Cabrera García et Montiel Flores, relative à l'arrestation et à la détention par des militaires de particuliers suspectés d'avoir commis des infractions pénales, et Vélez Loor, relative à l'arrestation et à la détention d'un migrant en situation irrégulière.

Elle rappelle dans la première affaire que cet article 7 contient deux types de normes: l'une générale, contenue dans son $1^{\mathrm{er}}$ alinéa, selon lequel «[t]oute personne a droit à la liberté et à la sécurité », et les autres plus spécifiques, les alinéas 2 à 7 prévoyant une série de garanties nécessaires à la protection de cette première. Par conséquent, la violation de l'une d'entre elles implique nécessairement une violation de l'article 7.1 de la Convention $(\$ 79)^{85}$.

Elle énonce ensuite la portée de ces différents alinéas, pris isolément. Nous examinerons dès lors successivement ces différentes garanties, à savoir le droit de ne pas être privé illégalement et arbitrairement de sa liberté (a), de connaitre les raisons de son arrestation et des accusations portées contre soi (b), le contrôle judiciaire de la privation de liberté (c) et la possibilité de contester la légalité de la détention $(\mathrm{d})$.

\section{Le droit de ne pas être privé illégalement et arbitrairement de sa liberté (articles 7.2 et 7.3)}

L'article 7.3 de la Convention impose que «nul ne peut faire l'objet d'une détention ou d'une arrestation arbitraires ». Concernant l'affaire Cabrera García et Montiel Flores, la Cour déduit de la violation de l'alinéa 5, qui impose aux États de traduire toute personne arrêtée ou détenue immédiatement devant un juge ${ }^{86}$, que la détention est devenue arbitraire. Mais c'est surtout dans l'affaire Vélez Loor qu'elle rappelle avec précision quelle est la portée à donner à cette garantie.

Elle réaffirme d'abord qu'elle recouvre une exigence de motivation, que l'État doit impérativement respecter, même si l'arrestation est justifiée par des raisons d'ordre public $^{87}$. La simple énumération, comme en l'espèce, de toutes les normes potentiellement applicables dans l'ordre de détention n'est pas suffisante au regard de cette exigence $(\$ 116)^{88}$. La Cour en profite pour souligner que le droit interne ne prévoit aucun délai de détention précis, laissant la possibilité pour les autorités administratives de la prolonger, la transformant de fait en mesure punitive $(\$ 117)^{89}$. Elle estime alors que l'État a violé l'article 7.3 de la Convention, combiné avec l'article 1.1 dans la mesure où la privation de liberté de 25 jours subie par la victime était fondée sur un ordre de détention qualifié d'arbitraire.

La Cour est ensuite confrontée à la question de savoir si les États ont la possibilité d'adopter une sanction pénale suite à une violation des lois relatives à l'immigration. Pour ce faire, elle rappelle la portée de l'article 7.2 de la Convention, qui impose que la législation interne prévoie de la manière la plus précise qui soit, et de manière anticipée, les causes et conditions d'une privation de liberté $(\$ 164)^{90}$, qu'elle relie avec l'article 7.3. Et pour déterminer si cette arrestation est arbitraire, elle se réfère à son «test de compatibilité» des restrictions des droits avec la Convention ( $\$ 165-167)$. Elle constate tout d'abord que la peine à laquelle a été soumise la victime - deux ans d'emprisonnement - a été "prévue par la loi» (\$ 167). Concernant ensuite la légitimité et le caractère approprié du but de la mesure ${ }^{91}$, la Cour souligne que les États ont la faculté de contrôler et de réglementer l'entrée et le séjour des étrangers sur leur territoire. La détention

83. La Cour renvoie alors aux affaires de la CEDH, Niemietz c. Allemagne, 16 décembre 1992, requête $\mathrm{n}^{\circ}$ 13710/88, $\$ 29$ et CEDH, Peck c. RoyaumeUni, 28 janvier 2003, requête $\mathrm{n}^{\circ} 44647 / 98, \$ 57$.

84. La Cour renvoie cette fois-ci aux décisions de la CEDH: M. C. c. Bulgarie, 4 décembre 2003, requête $\mathrm{n}^{\circ} 39272 / 98, \$ 150$, mais aussi du Tribunal international pour l'ex-Yougoslavie: Mucic et autres, "Celebici Camp», 16 novembre 1998, affaire n' IT-96-21-T, \$ 492.

85. Voir Cour IDH, Chaparro Álvarez et Lapo Íñiguez c. Équateur, exceptions préliminaires, fond, réparations et frais, 21 novembre 2007 , série C, $\mathrm{n}^{\circ} 170, \S 54$, ci-après «affaire Chaparro Álvarez et Lapo Íñiguez».

86. Voir infra.

87. Cour IDH, García Asto et Ramírez Rojas c. Pérou, exceptions préliminaires, fond, réparations et frais, 25 novembre 2005, série C, $\mathrm{n}^{\circ} 137, \$ 128$ et 143 .

88. La Cour a déjà affirmé que les décisions adoptées par les organes internes susceptibles d'affecter les droits, et qui ne sont pas correctement motivées, doivent être considérées comme arbitraires: affaire Yatama, $\$ 152$.

89. La Cour mentionne également le principe $\mathrm{n}^{\circ} 7 \mathrm{du}$ rapport du Groupe de travail du Conseil économique et social de l'ONU sur la détention arbitraire, annexe II, délibération $\mathrm{n}^{\circ}$ 5, 28 décembre 1999, E/CN.4/2000/4.

90. Voir aussi l'affaire Chaparro Álvarez et Lapo Íñiguez, $\$ 57$.

91. La Cour relève à cet égard que contrairement à la $\mathrm{CSDH}$, la Convention américaine ne prévoit pas de manière explicite quels sont les buts considérés comme étant «légitimes dans une société démocratique»; il lui revient dès lors la charge de les fixer elle-même. Affaire Vélez Loor, $\$ 168$. 
d'un migrant en situation irrégulière dans l'attente de procéder à son éloignement doit donc être considérée comme poursuivant un but légitime. Néanmoins, les États ne peuvent détourner cet objectif, et cette procédure ne peut ni déboucher sur des privations arbitraires de liberté ${ }^{92}$, ni transformer la détention en mesure punitive ${ }^{93}$, comme c'est pourtant le cas en l'espèce. Le but de cette restriction ne peut dès lors être considéré comme légitime. Quant à la nécessité de la mesure, la Cour rappelle que, «dans une société démocratique, le pouvoir punitif ne s'exerce que dans la mesure où il est strictement nécessaire à la protection des droits fondamentaux», sans quoi son exercice devient arbitraire ${ }^{94}$. Or, l'État a la possibilité d'utiliser des mesures moins restrictives mais tout autant efficaces afin de lutter contre l'immigration clandestine. La Cour constate au regard de tout ceci que l'État a violé l'article 7.3, lu de manière combinée avec l'article 1.1.

\section{Le droit de connaître les raisons de son arrestation et des accusations portées contre soi (article 7.4)}

La Cour rappelle dans les affaires Cabrera García et Montiel Flores et Vélez Loor qu'une personne arrêtée ou détenue doit aussitôt être informée, dans des termes simples et clairs, des raisons de son arrestation et des accusations portées contre elle ${ }^{95}$. Cette garantie a pour but d'empêcher toute détention illégale ou arbitraire, dès le début de celle-ci, et de protéger le droit de la défense de l'individu concerné ${ }^{96}$. Or, l'État n'ayant pas apporté la preuve que les victimes ont bien reçu une telle information, la Cour constate la violation de l'article 7.4 de la Convention.

\section{Le contrôle judiciaire de la privation de liberté (article 7.5)}

La Cour confronte l'arrestation et la détention des victimes des deux affaires avec les impératifs du $5^{\mathrm{e}}$ paragraphe de l'article 7 de la Convention, qui impose que les détenus doivent être traduits «dans le plus court délai devant un juge ou un autre fonctionnaire habilité par la loi à exercer des attributions judiciaires». Dressant, comme à son habitude, un bilan de sa jurisprudence antérieure, elle souligne l'importance du rôle du juge, qui se doit: «[de] garantir les droits des détenus, [d']autoriser l'adoption de mesures préventives ou coercitives lorsque cela est strictement nécessaire, et de rechercher, en général, si l'accusé est traité de manière compatible avec la présomption d'innocence, s'analysant comme une garantie contre la détention arbitraire ou illégale et du droit à la vie et l'intégrité personnelle ${ }^{97}$.

Dans l'affaire Vélez Loor, elle s'attarde sur la question de savoir si cette garantie est bien applicable, dans la mesure où la privation de liberté de la victime n'est pas liée à une infraction pénale mais uniquement à son statut de migrant irrégulier ${ }^{98}$. Soulignant la différence de rédaction entre les textes interaméricain et européen en la matière ${ }^{99}$, elle constate que ce premier ne limite aucunement l'exercice de cette garantie, qui est donc applicable quel que soit le motif de l'arrestation ou de la détention et répond donc par l'affirmative ${ }^{100}$.

Elle recherche dans un second temps si ladite disposition est respectée, tout en imposant aux États de prendre en considération la «situation de vulnérabilité particulière» dans laquelle se trouvent les migrants ${ }^{101}$. Elle précise alors la portée à donner à cet article 7.5. En ce qui concerne tout d'abord le «juge» ou le «fonctionnaire habilité par la loi à exercer des attributions judiciaires»,

92. La Cour se réfère pour justifier cet aspect au rapport Groupe de Travail des Nations unies sur la détention arbitraire, Promotion et protection de tous les Droits de l'homme, civils, politiques, économiques, sociaux et culturels, y compris le droit au développement, 10 janvier 2008, $\mathrm{A} / \mathrm{HRC} / 7 / 4, \S 53$. 93. La Cour se réfère pour justifier cet aspect au rapport Rodríguez Pizarro, $\$ 73$.

94. Cour IDH, Kimel c. Argentine, fond, réparations et frais, 2 mai 2008, série C, $\mathrm{n}^{\circ}$ 177, $\$ 76$. La Cour cite également le rapport du Groupe de travail du Conseil Économique et Social de l'ONU sur la détention arbitraire, Droits civils et politiques et, notamment, les questions de la torture et de la détention, 12 décembre $2005, \mathrm{E} / \mathrm{CN} .4 / 2006 / 7, \$ 63$

95. Affaires Vélez Loor, $\$ 171$; et Cabrera García et Montiel Flores, $\$ 106$. Cette information doit porter sur les faits mais aussi sur les principales bases juridiques sur lesquelles se fonde la détention. Voir affaire Chaparro Álvarez et Lapo Íñiguez, $\$ 71$.

96. Cour IDH, Juan Humberto Sánchez c. Honduras, exceptions préliminaires, fond, réparations et frais, 7 juin 2003 , série C, $\mathrm{n}^{\circ} 99, \$ 82$, ci-après "affaire Juan Humberto Sánchez».

97. Affaires Vélez Loor, $\$ 105$; et Cabrera García et Montiel Flores, $\$ 93$. La Cour fait ainsi référence aux affaires Tibi ( $\$ 114$ et 118$)$ et Juan Humberto Sánchez $(\$ 88)$. Elle rappelle à cet égard que «la privation de liberté ordonnée dans le cadre d'une procédure pénale devant les juridictions civiles ou militaires, comme mesure de précaution ou punitive, aboutissant à des détentions collectives ou programmées, et celles menées en dehors de toute légalité, constituent le premier acte avant de procéder à une exécution extrajudiciaire ou à la disparition forcée» : la Cour fait ainsi une synthèse de sa jurisprudence relative à l'article 7. Affaire Vélez Loor, $\$ 106$

98. La Cour relève aussi que la victime n'a pas demandé le statut de réfugié, ce qui lui permet d'exclure l'application des règles internationales relatives au droit d'asile: affaire Vélez Loor, $\$ 106$

99. La Cour relève, en effet, que d'après la CSDH, ce droit d'être aussitôt traduit devant un juge ne vise que les personnes arrêtées ou détenues dans les cas visés au paragraphe 1.c de l'article 5, à savoir «lorsqu'il y a des raisons plausibles de soupçonner qu'il a commis une infraction ou qu'il y a des motifs raisonnables de croire à la nécessité de l'empêcher de commettre une infraction ou de s'enfuir après l'accomplissement de celle-ci ». 100. Affaire Tibi, $\$ 118$.

101. Affaire Bayarri, $\$ 67$. La Cour se réfère également à la jurisprudence européenne: CEDH, Iwanczuk c. Pologne, 15 novembre 2001 , requête $\mathrm{n}^{\circ} 25196 / 94, \S 53$. 
elle rappelle qu'il doit répondre aux caractéristiques d'impartialité et d'indépendance telles qu'elles ressortent notamment de l'article 8.1 de la Convention ${ }^{102}$. De plus, cette autorité doit avoir le pouvoir de libérer la personne concernée si elle constate qu'elle fait effectivement l'objet d'une détention illégale ou arbitraire $(\$ 108)^{103}$. L'expression "doit être traduit devant un juge", ensuite, signifie que le détenu doit comparaître en personne, et qu'il doit être entendu personnellement $(\$ 109)^{104}$. Examinant la procédure interne, menée essentiellement par écrit, la Cour relève que l'État n'a pas apporté la preuve que la victime a été effectivement entendue, d'où une violation de cette disposition.

Dans l'affaire Cabrera García et Montiel Flores, la Cour s'attarde sur la condition de traduction « dans le plus court délai» devant un juge, garantie qui doit être observée avec encore plus d'acuité au vu du contexte de forte présence militaire sur le territoire concerné $(\$ 81-87)^{105}$. Elle en profite pour rappeler que les États doivent observer une extrême prudence lorsqu'ils utilisent les forces armées en tant qu'élément de contrôle social $(\$ 87)$ et respecter, autant que faire se peut, le principe de séparation entre les fonctions militaires et policières ${ }^{106}$. Ceci lui permet de signaler aux États à quelles conditions la faculté donnée aux forces armées de restreindre la liberté personnelle des civils peut être considérée comme compatible avec la Convention: il faut qu'elle satisfasse aux exigences de stricte proportionnalité applicable aux restrictions des droits, mais aussi à des critères d'exceptionnalité et de diligence $(\$ 89)$. Les victimes n'ayant été traduites devant les juridictions judiciaires que 5 jours suivant leur arrestation, le Mexique a enfreint cette garantie.

\section{La possibilité de contester la légalité de la détention (article 7.6)}

La Cour vérifie dans l'affaire Vélez Loor si la victime a eu accès à un recours effectif pour contester la légalité de sa détention, exigence posée par l'article 7.6 de la Convention ${ }^{107}$. Procédant à une interprétation littérale, la Cour insiste sur le fait que la question de la légalité de l'arrestation ou de la détention ne peut être soumise qu'à " une autorité judiciaire» - « un juge ou un tribunal» ${ }^{108}$, et qu'il s'agit là d' "une exigence fondamentale pour assurer un contrôle adéquat et minutieux des actes administratifs touchant aux droits fondamentaux» $(\$ 126)$. Or, en l'espèce, la victime n'a eu accès qu'au directeur national de l'Immigration et au ministre de la Justice, autorités qui ne peuvent être qualifiées comme telles $(\$ 127)$.

La Cour relève, en revanche, que d'autres voies de recours étaient ouvertes à l'époque des faits $(\$ 128)$, et que la victime a eu accès au Médiateur de la République. Elle va dès lors confronter ces aspects à l'exigence d'efficacité des recours, qui passe à la fois par la mise à disposition d'une assistance juridique - consistant en la rencontre avec un professionnel du droit ${ }^{109}$ - mais aussi par une information portant sur les possibilités d'accès à une représentation juridique gratuite. Le détenu, qui s'avère être de surcroît en situation de vulnérabilité aggravée du fait de son statut d'étranger ${ }^{110}$, n'a pas reçu une telle information, et le Médiateur de la République du Panama ne peut être considéré comme suffisant au regard de ces critères. Cet État a donc violé l'article 7.6 de la Convention, lu de manière combinée avec son article 1.1.

\section{B. Le droit de déplacement et de résidence (article 22)}

La Cour apprécie la portée de l'article 22 de la Convention dans les affaires Chitay Nech et Manuel Cepeda Vargas, portant toutes deux sur l'exil des membres de la famille des victimes respectivement vers la capitale du Guatemala et à l'étranger. Elle rappelle que cette liberté protège le droit d'entrée, de séjour et de quitter le territoire de l'État, mais aussi le droit de ne pas être déplacé en son sein même ${ }^{111}$. Il s'agit en outre d' « une condition indispensable au libre développement de l'individu ${ }^{112}$, et la jouissance de cette liberté ne peut en aucun cas être subordonnée à l'existence d'un but ou d'un motif particulier dont devrait justifier la

102. La Cour rappelle que ces garanties s'appliquent tant aux décisions des organes judiciaires qu'administratifs. Voir la Cour, Tribunal constitutionnel c. Pérou, fond, réparations et frais, 31 janvier 2001, série $C, \mathrm{n}^{\circ} 71, \$ 71$.

103. Voir également l’affaire Bayarri, $\$ 67$.

104. Voir également l'affaire Chaparro Álvarez et Lapo Íñiguez, $\$ 85$.

105. La Cour se réfère notamment aux Observations finales du Comité des Droits de l'homme des Nations unies, Colombie, 5 mai 1997, CCPR/C/79/ Add. $76, \$ 19$.

106. Cour IDH, Zambrano Vélez et autres c. Équateur, fond, réparations et frais, 4 juillet 2007, série $\mathrm{C}, \mathrm{n}^{\circ} 166, \$ 51$. Elle doit donc «limiter au maximum l'utilisation des forces armées en vue de contrôler la criminalité ordinaire ou la violence domestique». Voir également l'affaire Retén de Catia, $\$ 78$.

107. Elle rappelle la portée de cette disposition telle qu'elle ressort de sa jurisprudence antérieure, qui requiert que la personne détenue puisse avoir accès à un juge, qui va pouvoir examiner la légalité de la privation de liberté et, le cas échéant, ordonner sa libération. Cour IDH, El Hábeas Corpus Bajo Suspensión de Garantías (arts. 27.2, 25.1 y 7.6 Convención Americana sobre Derechos Humanos), opinion consultative n OC-8/87, 30 janvier 1987 , série $A, n^{\circ} 8, \$ 33$.

108. Article 7.6 de la Convention.

109. Cour IDH, Suárez Rosero c. Équateur, fond, 12 novembre 1997, série C, n $35, \$ 70$. La Cour estime, en effet, qu’à défaut, le droit à la défense du détenu se trouve limité. Cour IDH, Barreto Leiva c. Venezuela, fond, réparations et frais, 17 novembre 2009, série C, $\mathrm{n}^{\circ} 206, \$ 61-62$.

110. Affaires Vélez Loor, $\$ 128 ;$ Rosendo Cantú, $\$ 184$; et Fernández Ortega, $\$ 200$. Voir également l'affaire Communauté indigène Yakye Axa, $\$ 51$ et 63.

111. Affaire Chitay Nech, $\$ 139$; et Massacre de Mapiripán, $\$ 188$. La Cour se réfère également à la définition posée par la Commission des Droits de l'homme, Principes directeurs relatifs au déplacement de personnes à l'intérieur de leur propre pays, alinéa 2 de l'introduction, 11 février 1998 , E/ CN.4/1998/53/Add.2, ainsi qu'à tout un ensemble de normes internationales ayant pris acte de cette définition.

112. Affaire Manuel Cepeda Vargas, $\$ 197$. Voir Cour IDH, Ricardo Canese c. Paraguay, fond, réparations et frais, 31 août 2004 , série C, $\mathrm{n}^{\circ}$ 111, $\$ 115$ ci-après "affaire Ricardo Canese». 
personne concernée ${ }^{113}$. L'État a donc l'obligation d'adopter des mesures positives visant son exercice ${ }^{114}$, ainsi que de prévenir et de protéger d'éventuelles atteintes à ce droit, y compris vis-à-vis des tiers ${ }^{115}$.

Cette obligation de protection est examinée par la Cour dans l'affaire Chitay Nech. Elle souligne que le phénomène de déplacement interne, «en raison de [s]a complexité [...] et du large éventail de droits qu'elle affecte ou [qui risquent d'être enfreints] ", et au regard de «la situation de vulnérabilité spécifique dans laquelle se trouvent généralement les déplacés », est considéré comme étant «un facteur de facto » de non-protection ${ }^{116}$. Dès lors, même s'il n'est pas établi que le Guatemala ait formellement restreint la liberté de circulation des populations indigènes concernées, elle considère qu'il l'a malgré tout violée ${ }^{117}$.

La seconde obligation - de prévention - se voit attribuer une portée particulière dans cette même affaire. La Cour fait en effet référence à différents rapports d'experts pour souligner l'importance fondamentale qu'a «la connexion énergétique avec la terre» dans la culture maya, leur fuite impliquant « une grande perte culturelle et spirituelle» $(\$ 145)$. Ceci vient renforcer sa position traditionnelle, selon laquelle elle reconnaît que la relation qu'entretiennent les populations indigènes avec leurs terres est «indispensable au maintien de leurs structures culturelles et à leur survie ethnique et matérielle» $(\$ 147)^{118}$. Leur déplacement forcé en dehors de la communauté les place donc dans une «situation de vulnérabilité particulière», en ce qu'elle "crée un risque évident d'extinction culturelle ou physique» $(\$ 147)^{119}$. Les États doivent donc adopter des mesures de protection spécifiques afin de "prévenir et de renverser cette situation » $(\$ 147)$, et ainsi permettre leur retour au sein de la communauté, chose que le Guatemala n'a pas faite $(\$ 150)$.

La Cour souligne enfin dans les deux affaires que ce devoir de prévention doit s'accompagner d'une obligation de mener une enquête effective sur la violation des droits qui est à l'origine de ces déplacements, condition indispensable au retour "sécurisé et digne " ${ }^{120}$ des personnes concernées ${ }^{121}$. Se fondant sur tout un ensemble de normes internationales ${ }^{122}$, elle élargit cette obligation procédurale, qui n'était décelée jusque-là qu’à l'égard des exécutions extrajudiciaires, «l'absence d'enquête [en la matière pouvant] encourager ou perpétuer un exil ou le déplacement forcé » des personnes concernées ${ }^{123}$. Constatant que le Guatemala et la Colombie ont failli à cette obligation, la Cour en conclut qu'ils ont porté atteinte à l'article 22, lu conjointement avec l'article 1.1 de la Convention ${ }^{124}$.

\section{Les droits protégeant la démocratie}

La Cour constate dans l'affaire Manuel Cepeda Vargas que les droits politiques, la liberté d'expression et la liberté d'association jouent une «importance fondamentale» au sein du système interaméricain, en ce qu'elles ont toutes comme finalité «de rendre possible le jeu démocratique ${ }^{125}$. C'est pourquoi, elle opère une lecture combinée de ces trois articles (1). Mais elle les examine également de manière indépendante, comme l'attestent les affaires Chitay Nech et de la Guerrilha do Araguaia, dans lesquelles elle donne une portée particulière aux droits politiques (2) et à la liberté de pensée et d'expression (3).

\section{A. La lecture combinée des droits politiques (article 23), de la liberté d'association (article 16) et de la liberté de pensée et d'expression (article 13)}

La Cour rappelle dans l'affaire Manuel Cepeda Vargas que la Convention protège «les éléments essentiels de la démocratie», dont "l'accès au pouvoir» et «son exercice» $(\$ 172)^{126}$, et que ces trois dispositions s'inscrivent dans

113. La Cour se réfère au Comité des Droits de l'homme des Nations unies, Commentaire général n $n^{\circ}$ 27, 2 novembre 1999, CCPR/C/21/Rev.1/Add.9, $\$ 1,4$, 8 et 19, alors même qu'elle l'a déjà dégagé dans sa jurisprudence antérieure, notamment dans l'affaire Ricardo Canese, $\$ 138$, qu'elle cite par ailleurs. 114. Affaire Manuel Cepeda Vargas, $\$ 197$; et Massacre de Mapiripán, $\$ 170$.

115. Affaires Manuel Cepeda Vargas, $\$ 197$; et Chitay Nech, $\$ 141$. Voir affaire Valle Jaramillo, $\$ 139$; et Massacre de Mapiripán, $\$ 179$.

116. Affaire Massacre de Mapiripán, \$210.

117. Affaire Chitay Nech, $\$ 150$

118. Voir, entre autres, l’affaire de la Cour IDH, Communauté indigène Yakye Axa c. Paraguay, fond, réparations et frais, 17 juin 2005 , série C, $\mathrm{n}^{\circ}$ 125, $\S 135$.

119. Pour motiver cette approche, la Cour se fonde cette fois-ci sur le droit interne d'un État membre, à savoir la décision de la Cour constitutionnelle colombienne du 26 janvier 2009, Auto 004/009.

120. Affaire Chitay Nech, $\$ 149$

121. Affaire Chitay Nech, $\$ 149$; la Cour se fonde sur le principe $\mathrm{n}^{\circ} 28$ des Principes directeurs relatifs au déplacement de personnes à l'intérieur de leur propre pays, Commission des Droits de l'homme, 11 février 1998, E/CN.4/1998/53/Add.2.

122. La Cour cite, en effet, le rapport du représentant du secrétaire général sur les Droits de l'homme des personnes déplacées dans leur propre pays, Walter Kälin, 23 décembre 2010, A/HRC/16/43/Add.2, la Convention sur la protection et l'assistance des personnes déplacées, adoptée par l'Union africaine le 22 octobre 2009 à Kampala, mais aussi le Statut de Rome de la Cour pénale internationale du 17 juillet 1998.

123. Affaire Manuel Cepeda Vargas, $\$ 201$; principe déjà affirmé dans l'affaire Communauté Moiwana, $\$ 120$.

124. Affaires Chitay Nech, $\$ 149$; et Manuel Cepeda Vargas, $\$ 202$.

125. Affaire Manuel Cepeda Vargas, $\$ 171$; Cour IDH, Castañeda Gutman c. Mexique, exceptions préliminaires, fond, réparations et frais, 6 août 2008, série $\mathrm{C}, \mathrm{n}^{\circ} 184, \$ 140$. Elle cite également une décision de la Cour constitutionnelle de Colombie allant dans le même sens: déc. $\mathrm{n}^{\circ} \mathrm{C}-265$, M. P. Alejandro Martínez Caballero, 2 juin 1994.

126. Elle relève également l'article 3 de la Charte démocratique interaméricaine, adoptée par l'assemblée générale de l'OEA le 11 septembre 2001, allant dans le même sens. 
ce cadre. En ce qui concerne l'article 23, tout d'abord, elle constate qu'il protège le droit d'être élu, et qu'il impose à l'État de garantir les conditions nécessaires à la pleine réalisation ${ }^{127}$. La liberté d'expression, ensuite, assure la diffusion des informations ou des idées, même celles qui «paraissent ingrates à l'État ou à une fraction quelconque de la population ${ }^{128}$. Les États doivent s'abstenir d'agir d'une manière qui «favorise, encourage, promeut ou consolide» l'état de vulnérabilité ${ }^{129}$ dans lequel se trouvent certaines personnes à l'égard de cette liberté et adopter les mesures appropriées, nécessaires et raisonnables pour empêcher la violation des droits de ces personnes ${ }^{130}$. Elle rappelle enfin que la violation du droit à la vie ou l'intégrité personnelle imputable à l'État et qui a été motivée par l'exercice légitime de la liberté d'association ${ }^{131}$ peut générer une violation de l'article 16, qui protège le droit de s'associer à des fins politiques ${ }^{132}$. Elle ajoute, pour terminer, que «les voix de l'opposition sont indispensables dans une société démocratique ${ }^{133}$, puisque nécessaires au débat $(\$ 173)$, et que «la participation effective des individus, groupes et organisations et partis politiques d'opposition dans une société démocratique doit être garantie par les États ${ }^{134}$.

La Cour confronte ensuite ces principes généraux à l'espèce. Elle constate que les candidats de l'UP et du PCC se trouvent effectivement dans une situation de vulnérabilité ( $\$ 174)$. Même si le sénateur Cepeda Vargas, par ailleurs journaliste, a bien eu accès à des fonctions politiques, l'objectif de son assassinat était de l'en empêcher. La Colombie n'a dès lors pas rempli son obligation positive d'assurer les conditions nécessaires à l'exercice de cette fonction, et à sa participation dans le débat politique, d'où une atteinte aux articles 21 et 13 . Son activité a en outre été entravée par la violence exercée à l'encontre de son mouvement politique, fait qui est de nature à atteindre l'article 16. Dès lors, la Colombie a violé ces dispositions, relevant des «principes qui sont à la base de l'État de Droit et $[\ldots]$ du régime démocratique» $(\$ 177)$.

\section{B. Disparitions forcées et droits à la représentation politique} (article 23.1.a)

La Cour rappelle dans l'affaire Chitay Nech que les droits politiques ne sont pas seulement consacrés par la $\mathrm{CADH}$, mais qu'ils le sont aussi par d'autres instruments internationaux relatifs à la protection de la démocratie et du pluralisme ${ }^{135}$. L'article 23.1.a qui protège le droit à une participation politique effective et implique que toute personne ait la possibilité de "participer à la direction de sujets politiques", dans la mesure où il s'agit là du «moyen [...] dont disposent les démocraties pour garantir les autres droits prévus par la Convention» $(\$ 107)^{136}$. Or en l'espèce, la disparition forcée de la victime, maire d'une commune, a non seulement abouti à la suppression de ses droits politiques, mais l'a également empêché de mener son mandat à terme, et de participer à la formation des dirigeants de la communauté indigène dont il était issu. Soulignant la dualité du droit à la représentation $(\$ 115)$, la Cour considère qu'il s'agit dès lors d'une violation à la fois des droits politiques de la victime, mais aussi de ceux de la communauté $(\$ 113)$. Or cet aspect a une importance fondamentale: il s'agit d'un «prérequis nécessaire à la réalisation de questions [de société] telles que l'intégration, l'autodétermination et le développement des communautés autochtones dans un État pluriel et démocratique» (\$113). C'est pourquoi, l'État a l'obligation de garantir le droit des populations indigènes de «participer à la prise de décision portant sur des sujets et des politiques ayant potentiellement des incidences sur le droit ou le développement de ces communautés» et ce, en prenant en considération "leurs valeurs, usages, coutumes, rites et formes d'organisation» $(\$ 114)^{137}$. La victime en ayant été empêchée suite à sa disparition forcée, la Cour en conclut donc que le Guatemala a violé l'article 23.1.a de la Convention, lu de manière combinée avec l'article 1.1.

127. Affaire Yatama, $\$ 195$.

128. Cour IDH, Ríos et autres c. Venezuela, exceptions préliminaires, fond, réparations et frais, 28 janvier 2009, série C, $\mathrm{n}^{\circ} 194, \$ 105$, ci-après «affaire Ríos»; Cour IDH, Perozo et autres c. Venezuela, exceptions préliminaires, fond, réparations et frais, 28 janvier 2009, série C, $\mathrm{n}^{\circ} 195, \S 116$, ciaprès «affaire Perozo".

129. Affaires Ríos, $\$ 107$; et Perozo, $\$ 118$.

130. Affaires Ríos, $\$ 107$; et Perozo, $\$ 118$.

131. Cour IDH, Cantoral Huamaní et García Santa Cruz c. Pérou, 10 juillet 2007, série C, $\mathrm{n}^{\circ} 167, \$ 147$.

132. La Cour, Baena Ricardo et autres c. Panama, exceptions préliminaires, 18 novembre 1999, série C, $\mathrm{n}^{\circ} 61, \$ 156$.

133. La Cour se réfère également à la jurisprudence de la Cour européenne: CEDH, ÖZDEP c. Turquie, 8 décembre 1999, requête $\mathrm{n}^{\circ} 23885 / 94, \$ 41$; CEDH, Parti socialiste et autres $c$. Turquie, 25 mai 1998, requêtes $n^{\circ} 20 / 1997$ et 804/1007, $\$ 47$.

134. Affaire Yatama, $\$ 201$; avis sur la Condition juridique et droits des travailleurs migrants, $\$ 89$.

135. Se reporter au $\$ 107$ de l'affaire Chitay Nech.

136. La Cour cote tout un ensemble de normes de l'OEA y faisant référence.

137. Affaire Yatama, $\$ 225$. 


\section{Liberté de pensée et d'expression et droit à la vérité}

La portée de la liberté de pensée et d'expression est réaffirmée dans l'affaire de la Guerrilha do Araguaia ${ }^{138}$, dans laquelle la Cour va lier l'article 13 au traditionnel «droit à la vérité» ${ }^{139}$ inhérent aux articles 8 et 25 de la Convention $^{140}$. Pour ce faire, elle insiste sur le droit positif de rechercher et de recevoir des informations $(\$ 196-197)^{141}$. Elle souligne l'importance de cet aspect, la délivrance de l'information permettant «sa circulation dans la société, de manière à ce qu'elle puisse la connaître, y accéder et la valoriser ${ }^{142}$. Il s'avère dès lors indispensable, dans une "société démocratique», que les autorités soient régies par le principe de «divulgation maximale», selon lequel toute information est présumée accessible, et que cette liberté soit sujette à un régime strict d'exceptions $(\$ 199)^{143}$. Dès lors, dans le cadre d'une enquête, les victimes doivent pouvoir demander et recevoir des informations visant à établir la vérité ( $\$ 201)$. En l'espèce, le Brésil ayant tardé (six ans) à remettre certaines informations aux autorités chargées de l'enquête, et la juridiction interne ayant tenu pour leur examen une «audience secrète», la Cour estime que l'État s'est dès lors rendu responsable d'une violation de l'article 13, lu de manière combinée avec les articles 8, 25 et 1.1 de la Convention.

\section{Les droits protégeant les libertés relationnelles}

La Cour précise la portée de trois dispositions protégeant les libertés dites relationnelles: le droit à la personnalité juridique, le droit à la protection de la famille et le droit attaché à la catégorie particulière de personnes que sont les enfants. La Cour ne faisant que réaffirmer ses principes traditionnels concernant ces deux premiers droits ${ }^{144}$, nous nous contenterons ici d'analyser uniquement la protection accordée aux mineurs de l'article $19^{145}$. Cette disposition est appliquée dans les affaires Chitay Nech et Rosendo Cantú du fait de l'âge des victimes au moment des faits ${ }^{146}$, ainsi que dans l'affaire Communauté indigène Xákmok Kásek, alors même qu'elle n'identifie pas précisément les mineurs concernés, faisant uniquement référence à «tous les enfants de la communauté» ${ }^{147}$.

Elle rappelle la valeur de cette disposition, qui « doit être comprise comme un droit additionnel, complémentaire que le traité prévoit pour les êtres qui, par leurs développements physique et émotionnel, nécessitent une protection particulière ${ }^{148}$. L'État doit donc «adopter des mesures spéciales fondées sur le principe de l'intérêt supérieur de l'enfant » ${ }^{149}$, et " accorder une attention particulière à leurs besoins et à leurs droits, compte tenu de leur situation particulière de vulnérabilité ${ }^{150}$.

À partir de là, la Cour précise dans chaque affaire quelles sont les mesures de protection que l'État doit adopter, ces dernières « vari[a]nt selon les circonstances particulières de l'espèce et le statut personnel des enfants [en cause] ${ }^{151}$. Dans l'affaire Chitay Nech, la Cour crée une nouvelle obligation positive à la charge de l'État afin de

138. La Cour rappelle dans cette affaire les différents aspects de cette liberté, telle qu'elle ressort de sa jurisprudence traditionnelle: affaire de la Guerrilha do Araguaia, $\$ 96$ sq.

139. Voir L. Burgorgue-Larsen, «Commentaire $\mathrm{n}^{\circ} 25$ sous l'arrêt Bámaca Velásquez c. Guatemala ", in Les grandes décisions de la Cour interaméricaine des Droits de l'homme, p. 737-763.

140. La Cour considère en effet qu'il s'agit d'un droit inhérent au droit d'accès à la justice, qu'elle s'analyse en outre comme étant une forme de réparation; Cour IDH, Velásquez Rodríguez c. Honduras, fond, 29 juillet 1988, série C, $\mathrm{n}^{\circ} 4, \$ 181$.

141. La Cour considère en effet que cette liberté englobe «non seulement le droit d'exprimer ses pensées mais aussi le droit et la liberté de chercher, de recevoir et de diffuser des informations et des idées de tout genre"; Cour IDH, La Colegiación Obligatoria de Periodistas (art. 13 et 29 de la Convention américaine relative aux Droits de l'homme), OC-5/85, 13 novembre 1985 , série $\mathrm{A}, \mathrm{n}^{\circ} 5, \S 30$; et affaire Chaparro Álvarez et Lapo Íñiguez, $\$ 163$.

142. Cour IDH, Claude Reyes $c$. Chili, fond, réparations et frais, 19 septembre 2006, série C, $\mathrm{n}^{\circ} 151, \$ 77$, ci-après «affaire Claude Reyes". Ce droit contient dès lors deux dimensions - matérielle et sociale - qui doivent être garanties de manière simultanée par l'État: Cour IDH, Olmedo Bustos et autres ( $«$ La Última Tentación de Cristo ») c. Chili, 5 février 2001, série C, $\mathrm{n}^{\circ} 73, \$ 67$.

143. Voir aussi l'affaire Claude Reyes, $\$ 92$

144. Pour ce qui est de l'article 3, qui protège le droit à la personnalité juridique, la Cour ne fait que réaffirmer qu'il impose aux États de procurer des documents d'identité à ses ressortissants: affaire Communauté indigène Xákmok Kásek, \$251. Elle estime ensuite dans l'affaire Chitay Nech que les déplacements forcés de sa famille suite à sa disparition forcée ont entraîné une «désagrégation de la structure familiale» et que le Guatemala a donc violé l’article 17 de la Convention à leur égard: affaire Chitay Nech, $\$ 161$.

145. Pour une analyse complète de cet aspect, voir K. Martin-Chenut, «La condition juridique de l'enfant dans la jurisprudence interaméricaine des Droits de l'homme", Revue de science criminelle et de droit pénal comparé, ${ }^{\circ}$ 2, 2008, p. 416-428.

146. Pour ce qui est de la première, certaines victimes du déplacement forcé étaient âgées de moins de 18 ans au moment de la reconnaissance de la compétence contentieuse de la Cour par le Paraguay: affaire Chitay Nech, $\$ 166$. Concernant la deuxième affaire, la victime de violence sexuelle était également mineure au moment des faits: affaire Rosendo Cantú, $\$ 200$.

147. Affaire Communauté indigène Xákmok Kásek, $\$ 265$.

148. Affaires Chitay Nech, $\$ 164$; et Rosendo Cantú, $\$ 201$. Voir également l'avis Condición Jurídica y Derechos Humanos del Niño, opinion consultative $\mathrm{n}^{\circ}$ OC-17/02, 28 août 2002, série A, $\mathrm{n}^{\circ} 17, \$ 53,54$ et 60; et affaire Instituto de Reeducación del Menor, $\$ 147$.

149. Affaires Chitay Nech, $\$ 164$; Rosendo Cantú, $\$ 201$; et Communauté indigène Xákmok Kásek, $\$ 258$. Voir également l'avis Condición Jurídica y Derechos Humanos del Niño, $\$ 56$ et 6o. La Cour précise dans l’affaire Communauté indigène Xákmok Kásek que les effets de ce principe «irradient l'interprétation de tous les droits de la Convention lorsque l'affaire concerne un mineur» (\$258).

150. Affaire Masacre de las Dos Erres, exceptions préliminaires, fond, réparations et frais, 24 novembre 2009, série $C, \mathrm{n}^{\circ} 2111, \$ 184$.

151. Affaire Chitay Nech, $\$ 166$. 
prendre en considération la qualité d'indigène: le droit à la vie culturelle $(\$ 167)$. Pour ce faire, et ayant auparavant précisé que l'article 19 devait se lire à la lumière des Conventions américaine et internationale relatives aux droits de l'enfant ${ }^{152}$, elle s'appuie sur l'article 30 de cette dernière ${ }^{153}$, qui impose aux États de promouvoir et protéger le droit des enfants indigènes de vivre selon leur propre culture, leur religion, leur propre langue. Elle s'appuie également sur l'Observation générale $n^{\circ} 11$ du Comité des droits de l'enfant, qui estime que «l'exercice effectif de leur droit à leur propre culture, leur propre religion et leur propre langue constitue l'un des fondements essentiels d'un État pluriculturel», et que ce droit constitue « une reconnaissance importante des traditions et des valeurs collectives des cultures autochtones» $(\$ 168)^{154}$. Or la fuite des membres d'une communauté engendre une perte culturelle et spirituelle à l'égard des enfants, qui se retrouvent dans l'incapacité de recevoir un enseignement oral. De plus, «le développement des enfants est un concept holistique, qui englobe le développement physique, mental, spirituel, moral, psychologique et social ${ }^{155}$ et les enfants indigènes doivent «de préférence grandir et être éduqués dans leur environnement naturel et culturel », du fait de leur «identité propre qui les lie à leur terre, à leur culture, à leur religion, et à leur langue». Les enfants déplacés en dehors de leur communauté ont donc été privés de leur vie culturelle, et le Guatemala est responsable d'une violation des articles 19 et 1.1 lus de manière combinée.

Concernant l'affaire Rosendo Cantú, ensuite, la Cour prend soin de préciser la portée à donner à cette «obligation de protéger l'intérêt supérieur des enfants » et ce, "au cours de toute procédure», en prenant acte des différentes observations générales du Comité des droits de l'enfant de l'ONU. L'État doit dès lors: «i) fournir des informations et mettre en œuvre les procédures appropriées et adaptées à leurs besoins, veiller à ce qu'ils bénéficient d'une assistance juridique et autres, en tout temps ${ }^{156}$, ii) assurer, en particulier dans les cas où des enfants ont été victimes de délits, comme l'agression sexuelle ou d'autres formes de mauvais traitements, leur droit d'être entendu [...], en s'assurant que le personnel est formé pour les recevoir et que les lieux où il les rencontre soient sécurisés et qu'ils ne soient pas intimidants, hostiles, insensibles ou inadéquats ${ }^{157}$, et iii) faire en sorte que les enfants ne soient pas interrogés plus souvent que nécessaire, afin d'éviter, autant que possible, [leur] re-victimisation ${ }^{158} »(\$ 201)$.

Le Mexique n'ayant pas adopté de mesures spécifiques en faveur de la victime dans le cadre des procédures judiciaires menées au plan interne, alors qu'elle était mineure au moment des faits, a donc violé cette disposition (\$202).

Dans l'affaire Communauté indigène Xákmok Kásek, enfin, la Cour rappelle sa jurisprudence consultative selon laquelle l'éducation et la santé des enfants nécessitent diverses mesures de protection, et qu'elles constituent «les piliers fondamentaux pour assurer la jouissance d'une vie digne à l'égard des enfants » $(\$ 258)^{159}$. Or, en l'espèce, la Communauté a été privée d'un accès suffisant à l'eau, à la nourriture, à la santé et à l'éducation, éléments qui ont atteint tout particulièrement les enfants, victimes de malnutrition et de diverses maladies, ayant pour certains, entraîné leur décès ( $\$ 259-260)$. C’est pourquoi, le Paraguay s'est rendu coupable d'une violation des articles 19 et 1.1, lus conjointement. Mais la Cour constate également que ces dispositions sont violées sur la base d'un autre fondement: le droit à l'identité culturelle des enfants indigènes, tel qu'il ressort de la jurisprudence Chitay Nech ci-dessus analysée. En effet, la privation de la terre a entraîné, d'après la Cour, une perte des pratiques traditionnelles comme les rites d'initiation masculine ou féminine, mais encore la pratique des langues indigènes $(\$ 263)$.

La nouvelle formation de la Cour confirme ainsi les grandes tendances qui font la spécificité de la jurisprudence interaméricaine. Les références au jus cogens sont, en effet, réaffirmées, et la Cour s'attache toujours autant à imposer une protection accrue à l'égard des populations qualifiées de «vulnérables». Elle confirme enfin la méthodologie selon laquelle elle se fonde sur l'évolution $\mathrm{du}$ «corpus juris international» pour justifier l'interprétation donnée aux droits. On peut néanmoins relever une nouveauté notable, puisqu'elle s'appuie de plus en plus sur le droit interne des États membres de l'OEA. Ceci s'explique certainement par l'attitude récalcitrante de certains États, comme en atteste la décision du Supremo Tribunal Federal brésilien ${ }^{160}$ qui a affirmé la constitutionnalité de la loi d'amnistie alors même qu'il ressort de sa jurisprudence traditionnelle que ce type de législation

152. Voir avis Condición Jurídica y Derechos Humanos del Niño, \$ 194-196.

153. Convention internationale des droits de l'enfant, adoptée par l'assemblée générale des Nations unies le 20 novembre 1989 et entrée en vigueur le 2 septembre 1990. La Cour précise que le Guatemala a signé et ratifié cette Convention.

154. Comité des droits de l'enfant, Observation générale $n^{\circ} 11$. Les enfants autochtones et leurs droits en vertu de la Convention, 12 février 2009 , CRC/C/ $\mathrm{GC} / 11, \S 82$.

155. Comité des droits de l'enfant, Observation générale $n^{\circ} 5$. Mesures d'application générales de la Convention relative aux droits de l'enfant (art. 4 , 42 et 44, \$ 6), 27 novembre 2003, CRC/GC/2003/5, \$12, ci-après «Observation générale $n^{\circ} 5$ ". Ce concept de développement holistique a déjà été consacré par la Cour dans l'affaire Instituto de Reeducación del Menor, $\$ 161$.

156. Observation générale $n^{\circ} 5, \$ 24$; et Comité des droits de l'enfant, Observation générale $n^{\circ} 12$. Le droit de l'enfant d'être entendu, 20 juillet 2009 , $\mathrm{CRC} / \mathrm{C} / \mathrm{GC} / 12, \$ 64$, ci-après «Observation générale $n^{\circ} 12$ ".

157. Observation générale $n^{\circ} 12, \$ 21,34$ et 64 .

158. Observation générale $n^{\circ} 12, \$ 24$.

159. Voir avis Condición Jurídica y Derechos Humanos del Niño, $\$ 86$.

160. Supremo Tribunal Federal, Ação de Descumprimento de Preceito Fundamental (ADPF), nº 153, 29 avril 2010, disponible sur www.stf.jus.br. 
est incompatible avec la Convention ${ }^{161}$. Cette référence au droit interne des autres États qui en ont pris acte ${ }^{162}$ lui permet alors de réaffirmer sa position avec force ${ }^{163}$. Les nouveaux juges s'inscrivent donc dans la continuité de la «doctrine jurisprudentielle» interaméricaine ${ }^{164}$, que nous avons identifiée dans notre première chronique ${ }^{165}$, tout en l'enrichissant.

161. Affaire de la Guerrilha do Araguaia, $\$ 171$. Voir également Cour IDH, Barrios Altos c. Pérou, fond, 14 mars 2001, série $C$, $n^{\circ} 75, \$ 41$.

162. La Cour cite les décisions des cours suprêmes des États membres de l'Argentine, du Chili, du Pérou, de l'Uruguay et de la Colombie: affaire de la Guerrilha do Araguaia, $\$ 148-169$.

163. La Cour rappelle qu'en vertu de l'article 2 de la Convention, les autorités nationales doivent considérer que les normes de droit interne qui seraient contraires à l'objet et au but de la Convention n'ont aucun effet juridique. Cette disposition impose aussi au pouvoir judiciaire d'opérer un contrôle de conventionnalité ex officio pour y veiller et que le pouvoir judiciaire doit prendre en considération pour ce faire la Convention en tant que telle mais l'interprétation qui en est faite par la Cour: affaire de la Guerrilha do Araguaia, \$176.

164. García Ramírez Sergio, «Prologue», in Les grandes décisions de la Cour interaméricaine des Droits de l’homme, p. XXI.

165. M. Rota, «Chronique de jurisprudence de la Cour interaméricaine des Droits de l'homme», Cahiers de la recherche sur les droits fondamentaux, $\mathrm{n}^{\circ} 6,2008$, p. 182. 



\section{Liste des membres du Centre de recherche sur les droits fondamentaux et les évolutions du droit (CRDFED) (par ordre alphabétique)}

\author{
Tarek ABDAlla, Doctorant \\ Gilles Armand, Maître de conférences \\ Xavier Aurey, Doctorant (chercheur associé) \\ Maud BALdovini, Doctorante \\ Michel Bokouta, Doctorant \\ Sébastien Botreau-Bonneterre, Doctorant \\ Yann Butner, Doctorant \\ Servane CARPI-Petit, Maître de conférences \\ Maria Castillo, Maître de conférences \\ Aurore Catherine, Docteure \\ Agnès CERf, Maître de conférences \\ Catherine-Amélie Chassin, Maître de conférences \\ Dominique Custos, Professeure \\ Baptiste Deltaminil, Doctorant (chercheur associé) \\ Anne-Sophie Denolle, Doctorante \\ Édouard Des Courtils, Doctorant (chercheur associé) \\ Pauline Desert, Doctorante \\ Nathalie Deveze SANson, Doctorante \\ Samba Fall Dialo, Doctorant \\ Kossi Medoecho Dzreke, Doctorant \\ Sophie Elleni, Doctorante \\ Khaled El Zanati, Doctorant \\ Françoise ÉpINETte, Maître de conférences \\ Samuel ÉTOA, Maître de conférences \\ Oumar FofAnA, Doctorant \\ Lauréline Fontaine, Professeure (associée) \\ Grégory Godiveau, Maître de conférences \\ Boubacar Hassoumi Kountche, Doctorant \\ Nathalie Havas, Docteure
}

Sylvain Jacopin, Maître de conférences (associé)

Amoakon Koffi, Doctorant

Farida KorA, Doctorante

Alexandra Korsakoff, Doctorante

Christophe Lajoye, Maître de conférences

Thierry Lamulle, Maître de conférences

Jean-Manuel Larralde, Professeur

Juliette LeCAmE, Doctorante

Stéphane LeClerc, Maître de conférences Jean-Christophe Le Coustumer, Professeur Vincent Legrand, Maître de conférences

Séverine Leroyer, Docteure

Anselme Liboko, Docteur

Alberto Mendes, Doctorant

Hussam Mohammad, Doctorant

Habib Mouкoкo, Doctorant

Remy Bernard Ngombe, Doctorant

Rina RANDriamiarisoa, Doctorante

Marie-Joëlle Redor-Fichot, Professeure

Marie Rota, Doctorante

Élodie SaILlant, Maître de conférences

Vincent Souty, Doctorant

Aliou Sow, Doctorant

Christiantus TABI, Doctorant

Éléonore TAFOREL, Doctorante

Aurélie Tardieu, Maître de conférences

Jean-Jacques Thouroude, Maître de conférences

Céline Warnier de Wailly, Doctorante 


\section{Cahiers de la recherche sur les droits fondamentaux}

$\mathrm{n}^{\mathrm{o}} 1$ La garantie juridictionnelle des droits fondamentaux épuisé

$\mathrm{n}^{\mathrm{0}} 2$ Les titulaires particuliers des droits fondamentaux $\quad 15 €$

$\mathrm{n}^{\mathrm{o}} 3$ Surveiller et punir / Surveiller ou punir? $\quad 15 €$

$\mathrm{n}^{\circ} 4$ Quel avenir pour la laïcité cent ans après la loi de 1905? $\quad 15 €$

$\mathrm{n}^{\circ} 5$ L'enfant $15 €$

$\mathrm{n}^{\circ} 6$ Pouvoirs exceptionnels et droits fondamentaux $\quad 15 €$

$\mathrm{n}^{\circ} 7$ L'universalisme des droits en question(s).

$\mathrm{n}^{\circ} 8$ La liberté d'expression $\quad 15 €$

L'archive des Cahiers de la recherche sur les droits fondamentaux propose, en accès libre et gratuit, la totalité des articles publiés dans la revue, au format PDF, à l'exception des deux dernières années diffusées exclusivement sous forme de volumes imprimés : http://www.unicaen.fr/puc, rubrique «Archives en ligne». 


\section{CAHIERS DE LA RECHERCHE SUR LES DROITS FONDAMENTAUX}

\section{COMMANDE PERMANENTE}

à retourner aux Presses universitaires de Caen.

Un devis vous sera adressé à la publication du numéro.

\section{COMMANDE AU NUMÉRO}

$\mathrm{N}^{\mathrm{o}(\mathrm{s})}$ de la revue:

Pour plus d'informations sur les anciens numéros, consultez le site des PUC: www.unicaen.frlpucl

à retourner aux Presses universitaires de Caen, accompagnée de votre règlement par chèque libellé à l'ordre de: Agent comptable de l'Université de Caen.

Frais de port: France + Dom-Tom: $4 €$ pour 1 ex. $+1,50 €$ par ex. supplémentaire. Port étranger: nous consulter.

Nom, PrÉnOM:

Adresse :

Les numéros de cette revue sont également disponibles :

- chez votre libraire (Diffusion : AFPUD ; Distribution : Sodis)

- sur le site de vente en ligne aux particuliers du Comptoir des presses d'universités: $w w w . l c d p u . f r$

Presses universitaires de Caen

Esplanade de la Paix, Mrsh, 14032 Caen Cedex-France Téléphone: +33 (o)2 $31566220 \cdot$ Télécopie: +33 (o)2 31566225 Internet: www.unicaen.fr/puc·Courriel:puc@unicaen.fr 
